ANDRÉS DÍEZ-HERREROa

JUAN ANTONIO BALLESTEROS-CÁNOVAS ${ }^{\mathrm{b}}$

MAR GÉNOVAc

RAÚL PÉREZ-LÓPEZd

JULIO GARROTE REVILLA ${ }^{\mathrm{e}}$

\title{
CAMBIOS AMBIENTALES DETECTADOS POR DENDROGEOMORFOLOGÍA Y LIQUENOMETRÍA PARA EL ANÁLISIS DE AVENIDAS TORRENCIALES EN SISTEMAS FLUVIALES
}

\begin{abstract}
RESUMEN
El análisis de la frecuencia y magnitud de las avenidas torrenciales en los sistemas fluviales puede utilizar diferentes fuentes de datos: meteorológicas, hidrológicas, histórico-documentales, geológicogeomorfológicas y botánicas. Entre estas últimas, el potencial de la dendrogeomorfología para la reconstrucción de avenidas en sistemas fluviales torrenciales ha sido ya demostrado en la bibliografía especializada; sin embargo, los estudios que combinan otras técnicas de aproximación como la

a Departamento de Riesgos Geológicos y Cambio Climático, Instituto Geológico y Minero de España (IGME, CSIC). C/ Ríos Rosas, 23. 28003 Madrid. andres.diez@igme.es. ORCID 0000-0003-1106-191X.

b Departamento de Geología, Museo Nacional de Ciencias Naturales, Consejo Superior de Investigaciones Científicas.C/Serrano 115 bis.28006Madrid.juan.ballesteros@mncn.csic.es. https://orcid.org/0000-0003-4439-397X.

c Departamento de Sistemas y Recursos Naturales, E.T.S.I. Montes, Forestal y Medio Natural, Universidad Politécnica de Madrid (UPM). C/ José Antonio Nováis 10. 28040 Madrid. mar.genova@upm.es. https://orcid. org/0000-0003-1944-1888.

d Departamento de Riesgos Geológicos y Cambio Climático, Instituto Geológico y Minero de España (IGME, CSIC). C/ Ríos Rosas, 23. 28003 Madrid. r.perez@igme.es. https://orcid.org/0000-0002-9132-4806.

e Departamento de Geodinámica, Estratigrafía y Paleontología, Facultad de Ciencias Geológicas, Universidad Complutense de Madrid (UCM). C/ José Antonio Nováis 12. 28040 Madrid. juliog@ucm.es. https://orcid. org/0000-0002-7727-8014.
\end{abstract}

Fecha de recepción: 30-06-2021. Fecha de aceptación: 29-10-2021. 
liquenometría son comparativamente menos abundantes; y muy pocos estudios han cuestionado las ventajas y desventajas de aplicación de estas técnicas en estos ambientes fluviales tan particulares. Por ello, en el presente trabajo, además de discutir las ventajas y limitaciones del uso de la dendrogeomorfología y la liquenometría en el análisis de avenidas torrenciales, se muestran ejemplos de aplicación en diferentes sistemas fluviales para disponer de información complementaria e incluso alternativa a las fuentes clásicas.

El fundamento metodológico de las fuentes de datos botánicas se basa en que los cambios ambientales periódicos de carácter estacional generan en la flora una serie de elementos anatómicos que, en el caso de determinadas especies arbustivas y arbóreas en climas de estaciones contrastadas, se manifiestan en secuencias de anillos de crecimiento en raíces, troncos y ramas (estudiadas por la dendrocronología); y en el caso de determinadas especies de líquenes crustáceos, en el crecimiento progresivo de los talos (estudiadas por la liquenometría). Los resultados obtenidos son dataciones de eventos y mediciones de paleoniveles, área inundada, tiempo de permanencia, calados, velocidades y caudales generadores que pueden ser utilizados para el estudio de las avenidas torrenciales, lo que permite mejorar el análisis de frecuencias y la cuantificación de las magnitudes de avenidas pretéritas. Por otro lado, las variaciones interanuales de esos cambios ambientales (secuencias de años secos o húmedos, fríos o cálidos, variaciones en la insolación, plagas y epidemias, interferencia antrópica...) pueden ser una limitación a este uso en el análisis de avenidas, dado que requieren procesos previos de filtrado de la señal climática y ambiental para la identificación de las perturbaciones, mediante el establecimiento de series de referencia o patrón, o el desarrollo de curvas de crecimiento liquenométrico. Sin embargo, las ventajas y aportaciones de estas fuentes de datos y técnicas superan con creces las limitaciones metodológicas, lo que las convierte en un prometedor campo de estudio de los cambios ambientales en los sistemas fluviales.

Palabras clave: dendrogeomorfología; liquenometría; sistemas fluviales; avenidas torrenciales; inundaciones; cambios ambientales.

\title{
ENVIRONMENTAL CHANGES DETECTED BY DENDROGEOMORPHOLOGY AND LICHENOMETRY FOR THE ANALYSIS OF TORRENTIAL AVENUES IN RIVER SYSTEMS
}

\begin{abstract}
Flood frequency analyses relay on different source of data, including botanical records. Among these, the potential of dendrogeomorphology for flash flood reconstruction in torrential river systems has already been demonstrated in the specialized literature; however, studies combining other approximation techniques such as lichenometry are comparatively less abundant; and very few studies have questioned the advantages and disadvantages of applying these techniques in these particular fluvial environments. Here, we discuss the advantages and limitations of the use of Dendrogeomorphology and Lichenometry in the analysis of flash floods. We provide examples of application in different river
\end{abstract}


systems that show how to overcome the disadvantages and provide complementary and even alternative information to classical sources. The methodological basis of the botanical data sources is based on the periodic environmental changes that condition the growth rate of living trees, manifested in sequence of tree-rings (i.e. studied by Dendrochronology) as well as the growth of several crustaceous lichens' species, manifested in the accumulated centripetal growth of the stems (studied by Lichenometry). These growth sequences can be used for relative and absolute dating of flood events by identifying growth disturbances related to this process. The results obtained are event dating and measurements of palaeo-stage, flooded area, residence time, depths, velocities and generating flows that can be used for the study of flash floods, allowing to improve the analysis of frequencies and the magnitudes of past floods. Yet, several challenges remain when using these approaches. The interannual variations of these environmental changes (sequences of dry or wet, cold or warm years, variations in insolation, plagues and epidemics, anthropic interference...) could limit the use of these sources for analysing flash floods, given that they require prior filtering processes of the climatic and environmental signal for the identification of disturbances, through the establishment of reference or pattern series, or the development of lichenometric growth curves. However, the advantages and contributions of these data sources and techniques far outweigh the methodological limitations, making them a promising field for the study of environmental changes in river systems.

KeYwords: dendrogeomorphology; lichenometry; fluvial systems; flash floods; environmental changes.

\section{INTRODUCCIÓN}

En los sistemas fluviales naturales, los cambios ambientales tienen lugar con muy diferente frecuencia temporal y magnitud espacial, desde las variaciones infinitesimales del campo de velocidades del flujo acuoso, que se verifican cada segundo en cada punto del cauce fluvial y que afectan a dimensiones milimétricas, hasta las modificaciones geomorfológicas a escala de los valles fluviales, con rangos temporales que abarcan incluso millones de años. Entre ambos extremos se ubican los cambios ambientales con frecuencia temporal anual a secular y que afectan a tramos hecto- a kilométricos de una corriente fluvial, como las crecidas y avenidas extraordinarias del caudal circulante y sus efectos en el sistema fluvial.

Las avenidas torrenciales son flujos superficiales, normalmente bifásicos (agua y sólidos), que circulan como escorrentía concentrada, canalizados por los cauces de sistemas fluviales torrenciales, caracterizados por (Aristizábal et al., 2020): las elevadas pendientes en el perfil longitudinal del lecho; la rapidez de actuación de los factores desencadenantes, como la precipitación de alta intensidad, la fusión nival repentina, los deshielos súbitos o la rotura instantánea de almacenamientos o represamientos, que conllevan bajos tiempos de concentración y viaje de la onda del hidrograma de crecida, inferiores a 6 horas (avenidas súbitas o flash floods); un campo de velocidades con fuertes variaciones espacio-temporales y cambios del régimen de flujo entre supercrítico, subcrítico y frecuentes resaltos hidráulicos; y alta capacidad para erosionar, transportar y depositar carga sólida, tanto detrítica inorgánica (sedimento) como orgánica leñosa (troncos, ramas, tallos y raíces). Para el estudio de los cambios extraordinarios en estos sistemas fluviales, como son las avenidas 
torrenciales, y para la prevención de los efectos dañinos que producen a la sociedad -frecuentemente con pérdida de vidas humanas e importantes consecuencias económicas (Marchi et al., 2010)-, es fundamental disponer de bases de datos de eventos pretéritos, que nos permitan saber cuál ha sido su frecuencia y magnitud en el pasado, para tratar de inferir cuál puede ser su comportamiento en el futuro. Normalmente, estos catálogos de eventos y desastres pasados proceden de registros documentales (archivos, bibliotecas y hemerotecas) o de series instrumentales sistemáticas de medidas con aparatos (radares meteorológicos, disdrómetros, pluviómetros, estaciones de aforo...) que, en el mejor de los casos, permiten remontarse en el tiempo unas décadas o unos siglos. De ahí que eventos catastróficos del pasado en los sistemas fluviales, pero de muy baja frecuencia de ocurrencia (como avenidas de altos periodos de retorno y elevada magnitud) o alejados de los puntos de control, no queden registrados en las bases de datos instrumentales ni en los registros documentales y por lo tanto estos registros suelen estar sesgados e incompletos espaciotemporalmente. Es necesario extender esas series hacia el pasado y abarcando todo el territorio, tomando información de registros naturales en los que sí hayan quedado registrados esos eventos catastróficos o sus efectos (Díez-Herrero y García, 2016).

Entre los registros naturales donde quedan señalados los cambios ambientales pretéritos asociados a eventos de avenidas torrenciales en sistemas fluviales, que estudia una subdisciplina de las Ciencias de la Tierra llamada "Paleohidrología de inundaciones" o, más correctamente "Hidrología de paleoinundaciones" (Baker, 2008), se encuentran básicamente tres grandes conjuntos de fuentes de datos:

- Geológico-geomorfológico, que incluye los registros sedimentológicos (depósitos aluviales en zonas de flujo inefectivo o de alta energía, depósitos lacustres laminados, precipitados travertínicos y espeleotemas, etc.), y las geoformas erosivas (marcas, golpes de gubia, marmitas, canales incididos, etc.) y sedimentarias (canales de avulsión o crecida, barras e islas, lóbulos y cordones de derrame o desbordamiento, etc.).

- Hidrológico-glaciológico, que incluye los almacenamientos y flujos de aguas de avenida conservados en ríos, lagos, mares y océanos; y los registros en secuencias de hielo en glaciares y cuevas heladas.

- Biológico-botánico, que incluye los registros en las plantas (ecología de formaciones y especies de vegetación de ribera, y dendrocronología de las secuencias de anillos de crecimiento arbóreo y arbustivo) y en otros organismos simbióticos (liquenometría de líquenes crustáceos).

En el presente artículo se hace un repaso teórico y aplicado de las metodologías de estudio, ventajas y limitaciones para la detección de los cambios ambientales en los sistemas fluviales, para dos de las disciplinas que estudian sendos registros naturales aplicados al estudio de las avenidas torrenciales: la dendrogeomorfología y la liquenometría. Todo ello con el objetivo de cubrir la escasez de análisis críticos de estas disciplinas, al menos en la literatura científica en castellano e ilustrados con casos de estudio españoles. Especialmente novedosa es la recopilación de las técnicas liquenométricas aplicadas al estudio de las avenidas torrenciales, ya que no existen muchos trabajos publicados sobre ello, mucho menos en España y además que enumeren las aportaciones e incertidumbres de estas fuentes de datos. 


\section{Cambios ambientales detectados en la biota como fuente de datos PARA EL ESTUDIO DE AVENIDAS TORRENCIALES}

La detección de cambios ambientales a través de la información derivada de organismos vivos, como lo son las plantas y los líquenes, está basada en su propia condición de organismos sésiles, que los obliga a obtener la energía necesaria para vivir sin poder desplazarse. De hecho, estos organismos son testigos directos de los cambios ambientales que tienen lugar en su biotopo, como lo son los cambios geomorfológicos asociados a eventos extremos (Ballesteros-Cánovas y Stoffel, 2016). La utilización de las tasas de crecimiento anuales de dichos organismos puede ser empleada para la datación absoluta y/o relativa de los depósitos y las formas del terreno asociadas a la ocurrencia de eventos torrenciales en el pasado.

\section{Dendrogeomorfología de inundaciones}

La dendrocronología es la disciplina científica que se ocupa del análisis y la datación de los patrones de crecimiento de árboles, arbustos y herbáceas perennes que son capaces de formar anillos de crecimiento anuales o estacionales (Schweingruber, 2012). Especialmente en las zonas templadas del planeta, los cambios estacionales de temperatura y precipitación condicionan la actividad meristemática de las plantas leñosas (Aloni, 2015), favoreciendo la producción diferencial de células de madera temprana (en primavera y comienzos del verano) y células de madera tardía (a finales del verano y principios de otoño). La alternancia de madera temprana y madera tardía produce anillos concéntricos que representa el crecimiento anual/estacional de las plantas leñosas en un determinado lugar.

Aunque el crecimiento anual de las plantas leñosas está condicionado por factores internos (como la genética del individuo y su edad) y externos (como la temperatura, humedad, concentración de $\mathrm{CO}_{2}$ y características edafológicas), la ocurrencia de procesos naturales como aludes de nieve, avenidas torrenciales o deslizamientos (entre otros) pueden ocasionar daños en la vegetación que quedan registrados en los anillos de crecimiento (Stoffel et al., 2010a y b). Por ejemplo, el material transportado por estos procesos puede generar heridas, romper las ramas, exponer las raíces y enterrar o inclinar los fustes de los árboles que crecen en su zona de influencia. Cada una de estas perturbaciones induce una respuesta fisiológica en el árbol (Ballesteros-Cánovas et al., 2010a y b; Copini et al., 2015), lo que afecta a la tasa de crecimiento interanual. La dendrogeomorfología usa este tipo de perturbaciones en el crecimiento para identificar la ocurrencia de procesos naturales y reconstruir su actividad espacial y temporal (Alestalo, 1971).

En el caso particular de las inundaciones, el uso de las evidencias de origen botánico se remonta a mediados del siglo xx, cuando Robert Sigafoos realizó la primera descripción sobre el vínculo existente entre la vegetación ribereña y la frecuencia de las inundaciones a lo largo del río Potomac (Washington, EE. UU.) (Sigafoos, 1961 y 1964). En los últimos años, el empleo de las evidencias botánicas en el estudio de inundaciones se ha extendido a otras regiones del mundo, fundamentalmente para $(i)$ entender la variabilidad de los procesos hidrológicos en cuencas de montaña en relación con 
el cambio climático (Zaginaev et al., 2019; Ballesteros-Cánovas et al., 2018; Ballesteros-Cánovas et al., 2020; Ferrio et al., 2015; Therrell y Bialecki, 2014); (ii) definir probables escenarios y mejorar el análisis de frecuencia de inundaciones (Bodoque et al., 2020; Ballesteros-Cánovas et al., 2011a, 2016, 2017a y 2017b; Tichavský et al., 2020; Génova et al., 2015, 2018). Hoy en día, las evidencias botánicas son reconocidas como una fuente de información con una gran representatividad espacio-temporal (Diez-Herrero et al., 2013a y b; Bodoque et al., 2015), favorecida por la ubicuidad de la vegetación en zonas de montaña y la precisión anual de los anillos de crecimiento (Ballesteros-Cánovas et al., 2015a; Wilhelm et al., 2018a y 2018b).

Aunque existe una clasificación de evidencias botánicas para el análisis de inundaciones a escala de árbol completo (Diez-Herrero et al., 2007 y 2013a; figura 1), en la práctica se emplean fundamentalmente (figura 2):

i. Distribución de especies y edades: los procesos de sucesión vegetal en el fondo del valle y sobre los depósitos de inundaciones permiten que se pueda usar la edad/especie como proxy para delimitar la extensión de eventos pasados y asignar una edad mínima de los depósitos (Génova et al., 2015; Furdada et al., 2020; Fontana et al., 2021). Esta información puede emplearse para mejorar los mapas de peligrosidad de inundación y calibrar la extensión de las zonas afectadas derivada de los modelos hidráulicos.

ii. Heridas en los árboles: los daños en la corteza y tejidos cambiales generados por el impacto de la carga sólida transportada durante un evento de inundación produce heridas visibles en las partes del árbol más expuestas al flujo. Estas heridas generan cambios fisiológicos en la madera para evitar infecciones fúngicas (Shigo, 1984), que implican cambios en la tasa de crecimiento y tipología de células, tales como cambios bruscos en el crecimiento y existencia de tejidos caóticos, alteraciones físicas y químicas y formación de canales resiníferos traumáticos, que dependen de la especie vegetal, la edad y el estado fitosanitario del individuo (Ballesteros-Cánovas et al., 2015a). Los descortezados son considerados como altamente fiables para datar eventos torrenciales, y pueden ser utilizados como indicadores del nivel del agua en estimaciones de caudal circulante. Por lo general, los árboles tienden a cerrar dichas heridas, por lo que pasados unos años no son visibles desde el exterior, con lo que requieren muestreos dirigidos para su identificación.

iii. Árboles inclinados: durante inundaciones torrenciales, el árbol puede deformarse permanentemente si la presión hidrodinámica del flujo excede la elasticidad del fuste y del sistema radicular. Cuando esto ocurre, los árboles tienden a compensar dicha deformación generando madera de reacción (Timell, 1986), que se caracteriza por un crecimiento excéntrico y por cambios físico-químicos a nivel celular. En las coníferas, la madera de reacción se forma en el lado inclinado del tallo y se denomina madera de compresión. Sin embargo, en frondosas la madera de reacción se forma en el lado opuesto y se denomina madera de tensión. El anillo de crecimiento que muestra el inicio de esta respuesta excéntrica se puede usar para fechar el momento en el que ocurrió la inundación torrencial. Aunque los árboles inclinados no definen un nivel del agua alcanzada y por lo tanto no son indicadores de paleonivel, la deformación del fuste se 
ha asociado con la energía mínima de la corriente y, por lo tanto, indirectamente con el caudal circulante (Ballesteros-Cánovas et al., 2015c).

iv. Árboles enterrados: el transporte y posterior sedimentación de la carga sólida durante eventos torrenciales posibilita que se puedan encontrar en zonas de baja energía depósitos que están enterrando parcialmente a la vegetación existente. Los efectos y las consecuencias en el crecimiento del enterrado parcial de árboles dependen de distintos factores asociados a la edad del árbol, especies afectadas y profundidad y características edáficas de los depósitos. En algunos casos, el peso añadido de los depósitos torrenciales tiene un efecto en la actividad radicular, inhibiendo la división celular (Nanson \& Beach, 1977), lo que se traduce en una reducción significativa de la tasa de crecimiento del árbol (Friedman et al., 2005). A su vez, un depósito de detríticos finos y limos podría reducir el acceso de las raíces al agua disponible y nutrientes (Friedman et al., 2005), aunque depósitos ricos en nutrientes generan respuestas en el crecimiento opuestas (Mayer et al., 2010).

v. Árboles con raíces expuestas: la exposición de raíces ocurre cuando los bancos de orilla son erosionados durante eventos de inundaciones que superan el nivel de cauce lleno (Malik, 2008). Cuando esto ocurre, la raíz queda expuesta a cambios de temperatura más bruscos y a una mayor radiación solar. Esto hace que el crecimiento de la raíz se modifique, lo que genera un crecimiento excéntrico que lleva consigo un decrecimiento del tamaño de las células de la madera temprana y una mayor tasa de crecimiento de la madera tardía. Estos cambios son evidentes a escala macroscópica, aunque la datación precisa puede requerir un análisis microscópico, especialmente en raíces de especies de frondosas o con anillos muy estrechos (Ballesteros-Cánovas et al., 2017b).

vi. Árboles en zonas endorreicas: los árboles que crecen en áreas endorreicas pueden experimentar condiciones anóxicas prolongadas que inhiben el flujo y el transporte de las hormonas de crecimiento (como la auxina) e inducir cambios en la anatomía de la madera (Wertz et al., 2013). Estos cambios anatómicos pueden ser detectados en el registro anual de crecimiento y posibilitar la datación de este tipo de inundaciones.

Una de las mayores limitaciones para la reconstrucción dendrogeomorfológica de avenidas en ambientes fluviales es quizás que las secciones donde existe mayor probabilidad de encontrar evidencia coinciden con los tramos de mayor dinámica hidrogeomorfológica, por lo que en muchas ocasiones es difícil encontrar individuos arbóreos y arbustivos cuya edad se remonte más allá del último evento torrencial de gran magnitud. Este, junto con otros aspectos de las limitaciones de estos métodos en sistemas torrenciales, son descritos con mayor profundidad por Ballesteros-Cánovas et al. (2015a). 


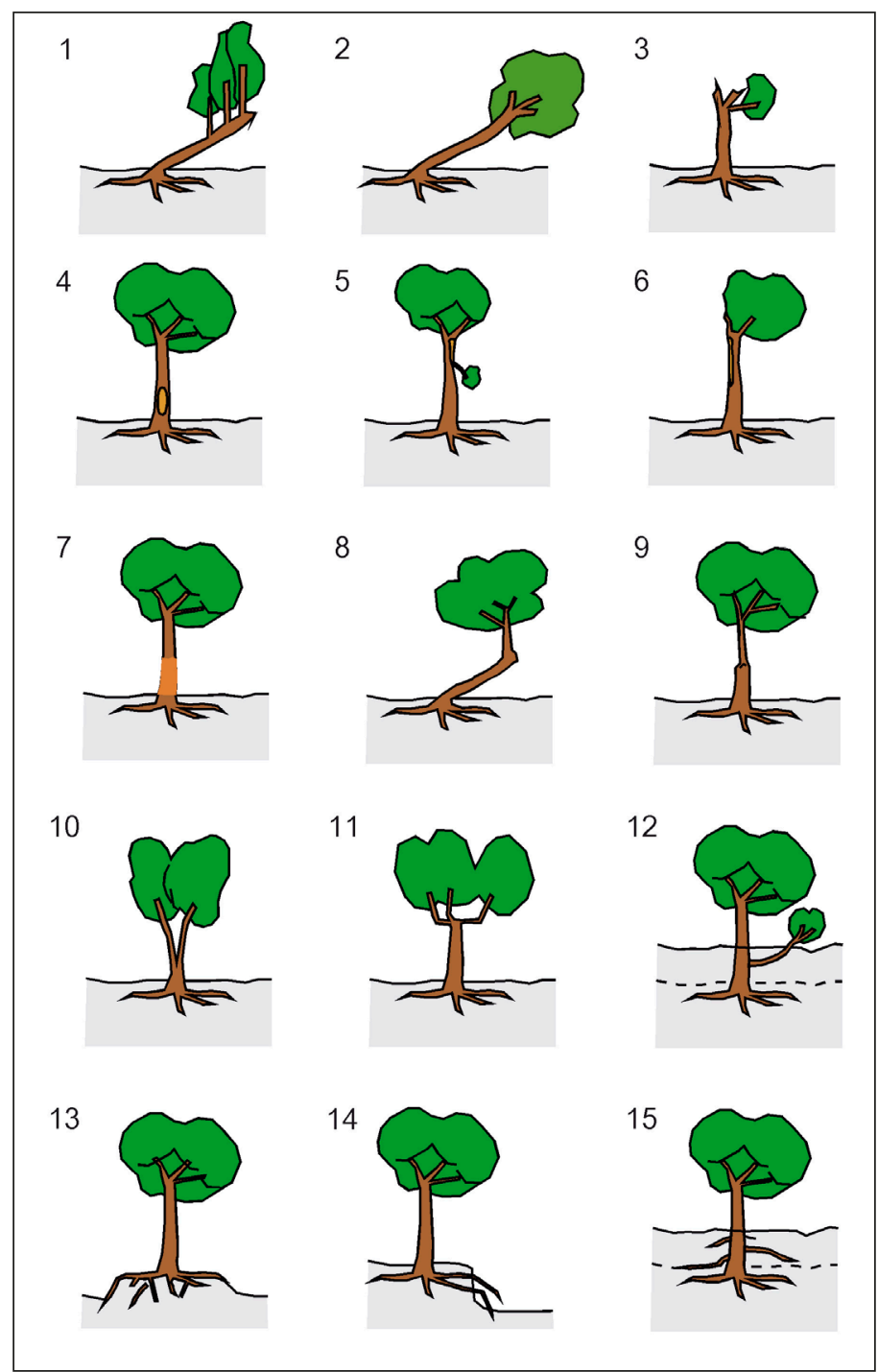

Figura 1. Principales tipos de evidencias dendrogeomorfológicas a escala de árbol completo, por la incidencia de las avenidas e inundaciones en el crecimiento del tronco, raíces o ramas de la vegetación arbórea, utilizadas para datar avenidas del pasado y estimar su magnitud (modificada de Díez-Herrero et al., 2007), representando un árbol: 1, inclinado y con rebrotes verticales; 2, inclinado; 3, decapitado; 4, con herida en la corteza del tronco (descortezado); 5, con arranque de ramas; 6 , con pérdida parcial de la copa; 7 , con bruñido de la corteza del tronco; 8 , con un codo brusco en el tronco; 9, con un estrechamiento brusco del tronco; 10, con un tronco bifurcado; 11, con ramas en candelabro; 12, con enterramiento de ramas; 13, con descalzado del cepellón en pedestal; 14, con raíces expuestas por descalce; y 15, con raíces adventicias tras enterramiento. 


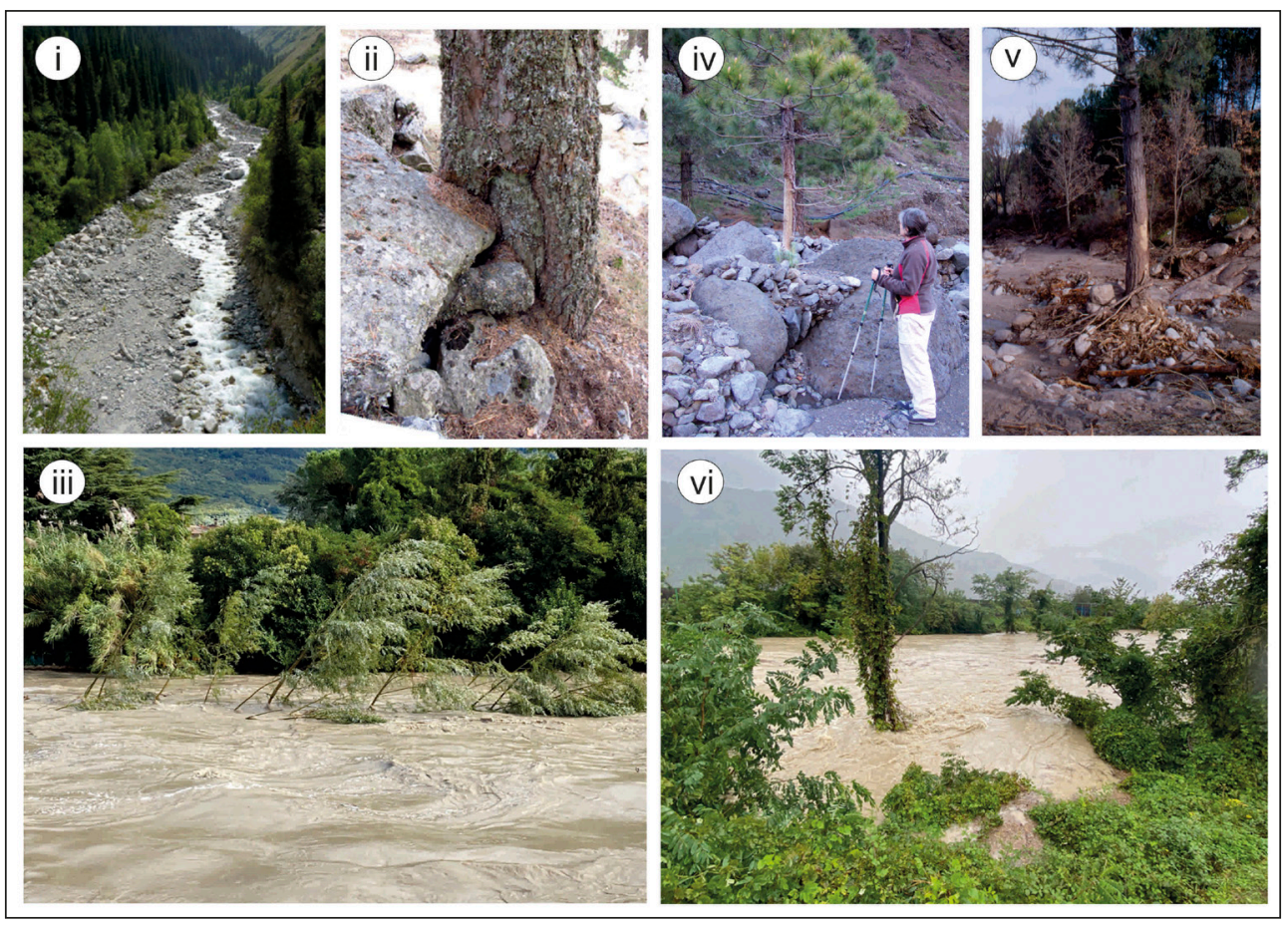

Figura 2. Ejemplos de los principales tipos de evidencias utilizadas en la práctica habitual del empleo de la dendrogeomorfología para el estudio de avenidas torrenciales: ( $I$ ) distribución de especies y edades; (ii) heridas en los árboles: los daños en la corteza y tejidos cambiales generados por el impacto de la carga sólida; (iii) arboles inclinados; (iv) árboles enterrados; ( $v$ ) árboles con raíces expuestas; (vi) árboles en zonas endorreicas.

Por lo general, el análisis de los anillos de crecimiento se realiza en testigos extraídos con una barrena de Pressler, aunque en árboles muertos o en raíces también se suelen extraer secciones o cuñas de madera (figura 3). En el muestreo, la selección del árbol es fundamental para minimizar el posible ruido en la señal asociado a otros factores abióticos. El muestreo debe ser dirigido a zonas del cauce que presenten una mayor energía en el flujo (Ballesteros-Cánovas et al., 2016; Génova et al., 2018; Victoriano et al., 2018; Quesada-Román et al., 2020a), recopilando información adicional asociada tanto del árbol (especie, altura, diámetro) como de su posición geomorfológica respecto del cauce. Una vez realizado el muestreo, los testigos y las muestras deben ser preparadas para su análisis, lo que incluye el secado de la madera, su lijado y posterior pulido para una mejor identificación de los anillos de crecimiento. La etapa de análisis conlleva la medición y datación de los anillos por medio del cofechado (Douglass, 1941; Fritts, 1976) y la identificación posterior de las anomalías de crecimiento asociadas a las evidencias reconocidas. 
Para que pueda elaborarse una secuencia continua de datos referidos a los anillos de crecimiento, es imprescindible que estos se produzcan de manera regular cada año y que presenten límites definidos. Esto sucede, generalmente, cuando el clima es estacional. Sin embargo, en ocasiones se producen anomalías que conducen a incertidumbres en la datación. Las más habituales suceden, en primer lugar, cuando la formación de la estructura xilemática (madera) se detiene y se reinicia más de una vez al año y se forman complejos de anillos. Estos se denominan anillos falsos, anillos dobles, zonas de crecimiento, anillos intraanuales o anillos con fluctuaciones de densidad intraanual, y se producen, por ejemplo, en climas tropicales con escasa estacionalidad, o por eventos de extrema aridez o heladas tardías, o por defoliaciones originadas por incendios o ataques de patógenos (De Micco et al., 2016). En otros casos se forman anillos incompletos, discontinuos, debido a condiciones de crecimiento extremas, defoliaciones o pérdida parcial de la copa o elevada edad (Schweingruber, 2007) o no se constituyen en absoluto (anillos ausentes). Mientras que los primeros a menudo se pueden cuantificar mediante análisis anatómicos detallados de la madera, los segundos se tratan como características cualitativas o como variables binarias (presente/ausente) que dificultan la datación precisa, pero que también aportan información sobre eventos que afectaron al crecimiento (Bräuning et al., 2016). Existen trabajos específicamente focalizados en la información climática y ecológica que puede obtenerse a partir tanto de los "falsos anillos" (más apropiado denominarlos "fluctuaciones en la densidad de la madera", IADF) y de los anillos ausentes en la Península Ibérica (Novak et al., 2013, 2016a y 2016b; Zalloni et al., 2016) ¿Cómo solventar estas anomalías en la datación precisa de los anillos de crecimiento? En dendrocronología se utilizan distintas técnicas que, mediante la comparación visual y estadística de los patrones de las secuencias de anillos de diferente origen, permiten asegurar la datación correcta de cada uno de los valores que integran las secuencias. Este complejo proceso, denominado interdatación o datación cruzada, es uno de los pilares o principios de esta disciplina y ya fue reconocido y utilizado por Douglass (1941).

Por lo general, la medición de los anillos se lleva a cabo por medio de mesas de medición o por el análisis de las imágenes escaneadas usando aplicaciones informáticas de procesamiento específico. La cronología de eventos final dependerá del número de anomalías identificadas para cada año, así como del número de muestras analizadas. Para ello, se pueden emplear índices de ponderación y umbrales de detección para aceptar o rechazar la existencia de un evento. En el caso de que las evidencias botánicas definan niveles de aguas o paleonivel (Baker, 2008), las alturas de estas marcas son usadas para calcular indirectamente el caudal necesario para alcanzar dicho nivel usando ecuaciones empíricas o modelos hidráulicos (Benito et al., 2015; Victoriano et al., 2018). Finalmente, los eventos de inundaciones reconstruidos por medio de evidencias botánicas pueden ser incluidos en el análisis de frecuencia (Génova et al., 2015, 2018; Bodoque et al., 2020; Ballesteros-Cánovas \& Álvarez-Troncoso, 2021), lo que mejora la estimación de cuartiles y reduce las incertidumbres asociadas. 


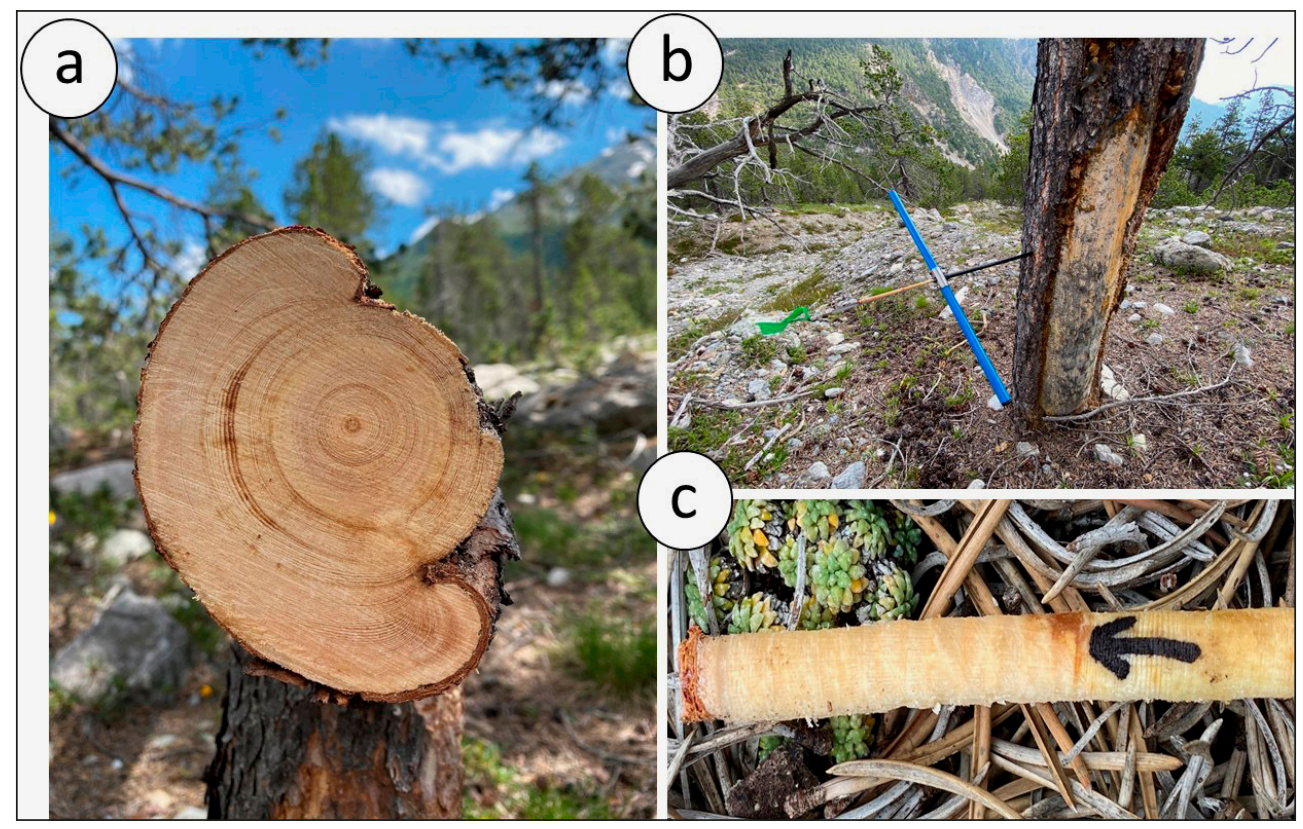

Figura 3. Muestreos dendrocronológicos en un parque nacional suizo: a) Sección transversal de un árbol herido por el impacto de la carga solida durante eventos torrenciales, $b$ ) fuste del árbol con barrena de Pressler de un árbol dañado; y c) señal característica de una herida en un testigo extraído con barrena de Pressler.

Fuente: Ballesteros-Cánovas y Álvarez-Troncoso (2021).

\section{Liquenometría en sistemas fluviales}

La liquenometría trata del estudio del tiempo de exposición de superficies rocosas a partir de la determinación de la edad de los líquenes que crecen sobre dichas superficies (Innes, 1986). Es por ello que esta técnica de datación a partir del estudio de los líquenes es aplicada en eventos geomorfológicos y de modelado del paisaje (figura 4). Para ello, es necesario determinar en primer lugar la especie de liquen o liquen reloj (Pérez-López et al., 2019), generalmente un liquen incrustante que aparece tanto sobre la superficie rocosa que queremos datar como en aquellos elementos utilizados para establecer el crecimiento anual (mm/año). La especie de liquen más utilizada en liquenometría es Rhizocarpon geographicum (L.) DC., debido a su amplia distribución en cualquier tipo de clima y sistema, siempre asociado a rocas ácidas y siliciclásticas, así como a sus tasas de crecimiento lentas y constantes (Bradwell \& Amstrong, 2006; Amstrong 2016). También se usan otras especies de líquenes sobre rocas básicas, en general sobre calizas y dolomías, como Aspicilia calcarea (L.) Körb., y sobre superficies artificiales cementadas, como Lecanora muralis (Schreb.) Rabenh. (Pérez-López et al., 2012). 


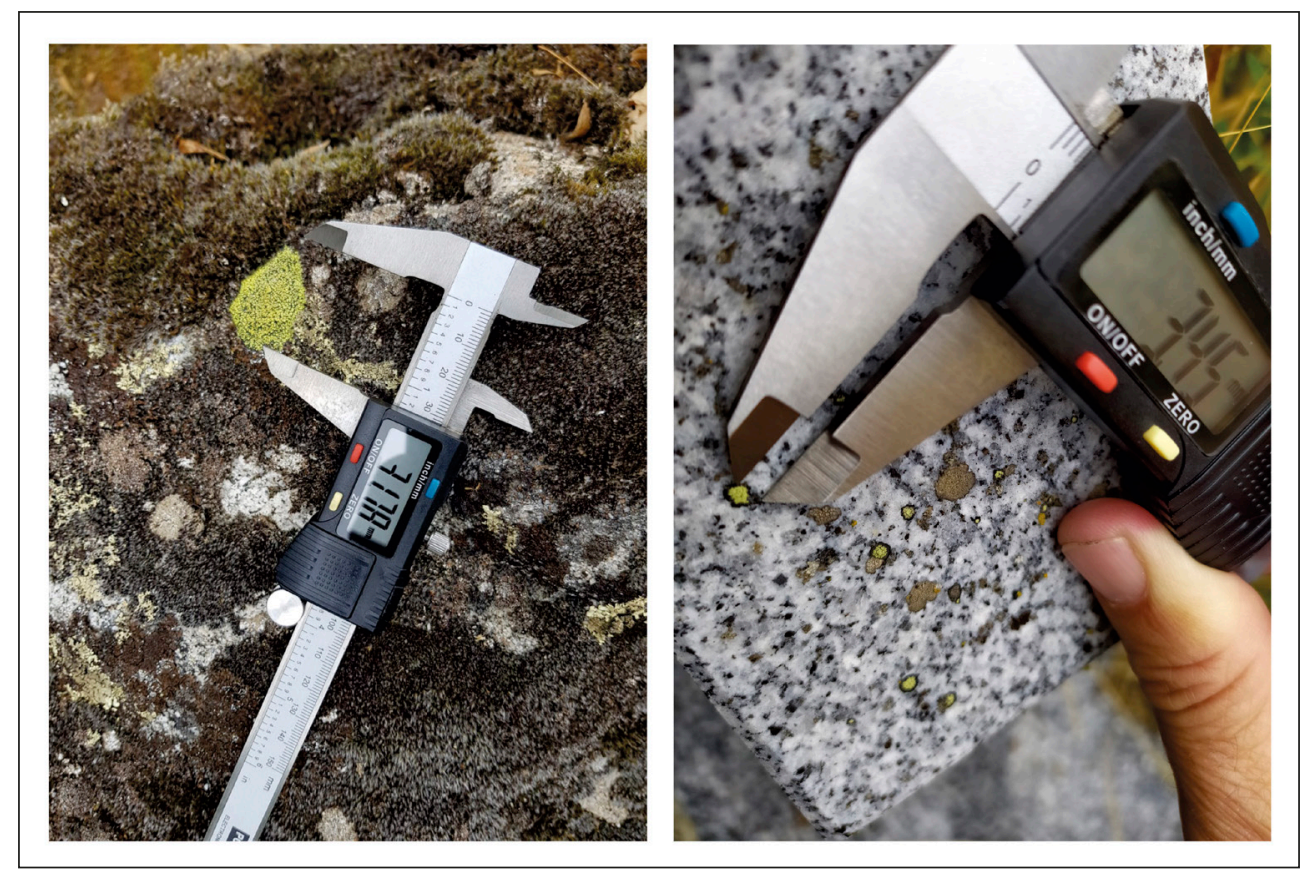

Figura 4. Talos del liquen Rhizocarpon geographicum (L.) DC. subsp. geographicum medidos con un calibrador digital sobre bloques graníticos de un lóbulo de flujo de derrubios tras una antigua avenida torrencial en la Sierra de Gredos (izquierda) y en la cruz de una sepultura en un cementerio próximo, en la provincia de Ávila (derecha), para el establecimiento de la curva de crecimiento liquenométrico.

Fuente: Díez-Herrero (2021).

Sin embargo, la ausencia de un protocolo ampliamente aceptado por la comunidad geomorfológica en la obtención de edades liquen deja muchas dudas sobre ciertos trabajos y sus resultados (Osborn et al., 2015; Rosenwinkel et al., 2015). En este sentido, uno de los puntos clave en la adecuada utilización de la liquenometría es la correcta determinación de la tasa de crecimiento y su extrapolación hacia atrás en el tiempo. Se recomienda no aplicar la liquenometría en intervalos de tiempo que vayan más allá de la edad del liquen más antiguo conocido de esa especie en las condiciones ambientales de estudio, realizar una estimación de errores de medida y ajustar la curva de crecimiento con varias funciones (Pérez-López et al., 2019).

En cuanto al uso de la liquenometría para el estudio de sistemas fluviales, varios trabajos se han centrado en el estudio de la dinámica fluvial de transporte sedimentario (Gob et al., 2003), estudiando el depósito de grandes bloques; así como los cambios de incisión en cauces encajados en roca (figura 5) mediante el uso de Rhizocarpon geographicum; o la edad de las diferentes terrazas fluviales, y determinaron que las últimas correspondían a la Pequeña Edad de Hielo (PEH). En este caso, dichos autores 
lograron calibrar una curva de crecimiento hasta 2.000 años atrás, aunque solo los últimos 400 años muestran errores aceptables para poder datar.

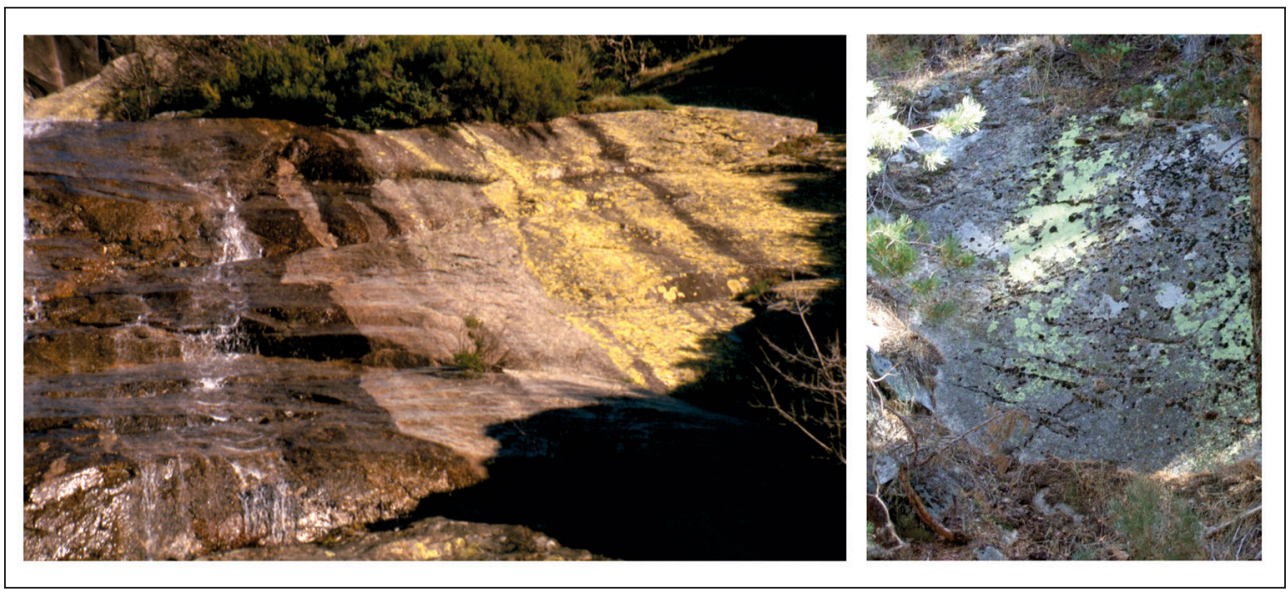

Figura 5. Superficies rocosas cubiertas por talos de líquenes de densidades y tamaños zonados de acuerdo con la frecuencia y magnitud de los eventos de avenida torrencial que las alcanza: lecho del arroyo de El Chorro Grande en Palazuelos de Eresma (izquierda) y margen izquierda del arroyo de Los Puentes en los Montes de Valsaín (derecha); ambas en la provincia de Segovia.

Fuente: Díez-Herrero (2021).

\section{CASOS PRÁCticos DE APLICACIÓN}

Para ilustrar el uso de la dendrogeomorfología y la liquenometría para el análisis de las avenidas torrenciales se han seleccionado tres casos de estudio en territorio español, escogidos entre los múltiples trabajos publicados con el criterio de que resulten representativos: de las ventajas y limitaciones de estas fuentes de datos y técnicas, y de su grado de utilización a nivel internacional (dos dendrogeomorfológicos puros y uno mixto dendrogeomorfológico-liquenométrico); de diferentes ámbitos geográficos, con dos en zonas de montaña de la Península Ibérica (Sistema Central español y Pirineos) y uno en relieves volcánicos de las islas Canarias y de que aborden diferentes objetivos de análisis, como son la evaluación del riesgo de inundación en una colonia infantil de veraneo, la prevención de daños a infraestructuras de transporte y ocio, y la gestión del uso público en un espacio natural protegido.

\section{Barranco de las Angustias en el P.N. de la Caldera de Taburiente}

La Caldera de Taburiente ocupa 4.690 ha del sector centro-septentrional de la isla de La Palma (Islas Canarias), y fue declarada Parque Nacional en el año 1954. Presenta un rango altitudinal entre los 2.426 m s.n.m. del Roque de los Muchachos y los $430 \mathrm{~m}$ del fondo del barranco de Las Angustias, a 
su salida del Parque Nacional. El estudio dendrogeomorfológico de avenidas torrenciales se centró en un tramo del fondo aluvial-coluvial del barranco de Taburiente, desde la confluencia de sus tributarios (los barrancos Verduras de Alfonso y Cantos de Turugumay), hasta su paso por las inmediaciones del Centro de Servicios del Parque.

Se trata de una llanura aluvial formada por grandes barras de bloques y gravas entre las que discurren uno o varios hilos de corriente, con una terraza baja separada por un escarpe de banco de orilla de unos 2,3 m de altura media. Las barras estabilizadas y las terrazas presentan vegetación arbórea de pino canario (Pinus canariensis Chr. Sm. ex DC.) y sauce canario (Salix canariensis C. Sm. ex Link). En esta zona convergen buena parte de los senderos más transitados y se encuentra la única zona de acampada autorizada, estimándose una población expuesta anual media en tránsito por el sistema de barrancos en unas 67.000 personas (A. Palomares, com. per.).

En estos barrancos son frecuentes e intensas las avenidas súbitas, las cuales han producido incluso víctimas mortales entre practicantes de senderismo (tres fallecidos en noviembre de 2001); varias situaciones de movilización de medios de protección civil al quedar grupos numerosos de visitantes incomunicados por la inundación (la última el 22 de octubre de 2011); e importantes pérdidas económicas al interferir las avenidas con los proyectos de repoblación de las riberas con especies autóctonas y endemismos (superiores a los 700.000 euros en los últimos años) e infraestructuras (puentes, conducciones de agua, sistemas hidroeléctricos) y las sendas más transitadas.

Ante la imposibilidad de realizar análisis de peligrosidad con métodos hidrológico-hidráulicos convencionales, por no existir estaciones de aforo representativas ni datos pluviométricos con series largas y discriminación espacial y temporal adecuada, se ha recurrido a los métodos dendrogeomorfológicos para la datación de eventos de avenidas. En total se muestrearon 73 ejemplares de Pinus canariensis mediante barrena de Pressler y, además, se obtuvieron varias cuñas y secciones discoidales con motosierra mecánica en tres ejemplares muertos y en un resto de madera subfósil localizado en el relleno de la terraza. Al menos se extrajeron, en los árboles afectados (con heridas producidas por la carga sólida transportada durante las riadas y/o con otros daños como desenraizamiento, pérdida de la guía principal, etc.), dos muestras completas por árbol (desde la corteza hasta la médula). Otras muestras se extrajeron cerca de las heridas, de manera que el contraste entre las diversas secuencias de crecimiento permitiera identificar el año en el que se produjo el daño. También se extrajeron muestras de dieciséis árboles no dañados para elaborar una cronología de referencia (Génova et al., 2015).

A partir del análisis de las secuencias de crecimiento en grosor de los árboles afectados se ha podido reconstruir el registro reciente (últimos cincuenta años) de los eventos de avenidas torrenciales que han sufrido estos barrancos. Se han datado sesenta daños en ocho años diferentes, y son especialmente relevantes los producidos en 1962 y 1997, pues se repiten sistemáticamente en muchos de los ejemplares muestreados (en el 48 \% y el $22 \%$, respectivamente, de los árboles dañados), mientras que en el resto de los años identificados $(1979,1993,2001,2003,2007$ y 2009) los porcentajes de replicación son inferiores al 15 \% (figura 6), lo que aporta información acerca de la magnitud y extensión de las avenidas (Génova et al., 2015). 
A raíz de la elevada incertidumbre en la morfología de lecho fluvial asociado al evento de 1962 (por los procesos de erosión-deposición, e incluso ensanchamiento del cauce), debido al tiempo transcurrido hasta la actualidad, se ha procedido a calibrar un modelo hidráulico para la zona, a partir de la información dendrogeomorfológica recopilada y asociada al evento de 1997. Para este evento se han considerado dos posibles morfologías de la superficie del lecho fluvial: por un lado, la denominada v1997 (derivada de la posición de los árboles afectados) y la altura de su base; y por otro lado, la denominada como v2009 (por la fecha en la que se recopilaron los datos topográficos en los que se basa el MDT de la zona). Así, para este evento, el caudal punta líquido estimado se sitúa en $1.235 \mathrm{~m}^{3} / \mathrm{s}$ (Garrote et al., 2018a), con un valor de RMSE frente a las alturas de los descortezados de 0,20 m; valor de caudal semejante al mejor ajuste obtenido para la topografía v2009 (RMSE de 0,3 m), y que plantea un posible escenario de compensación entre los fenómenos de erosión y sedimentación a lo largo de un evento de avenida. Este valor de caudal punta excede los resultados de los múltiples modelos hidrológicos derivados del registro de precipitaciones disponible en la zona (y de las tareas de maximización de lluvias por concentración temporal, y aplicación de gradientes altitudinales, llevadas a cabo; Garrote et al., 2018b). Situación esta última que pone de manifiesto la deficiente información pluviométrica disponible en la zona.

De este modo, la información aportada por los datos dendrogeomorfológicos supone una importante mejora para el desarrollo de los análisis de peligrosidad por inundaciones en la zona, que nos muestran un claro cambio en el patrón de zonas inundadas y de las características de flujo (velocidad y profundidad de la lámina de agua, principalmente) asociadas. Estos cambios se traducen en variaciones muy significativas de las zonas de elevada peligrosidad por inundaciones (Garrote et al., 2018a), y principalmente de la peligrosidad asociada al arrastre de personas por pérdida de estabilidad por la velocidad y el calado de la corriente. Así, frente a un patrón multicanal separado por grandes barras expuestas (que se asociaría a la modelización hidráulica de los caudales punta derivados a partir del registro de precipitaciones en la fecha de ocurrencia de evento analizado), el modelo hidráulico calibrado mediante información dendrogeomorfológica muestra un patrón fluvial con canal principal y varios canales secundarios bien desarrollados. 

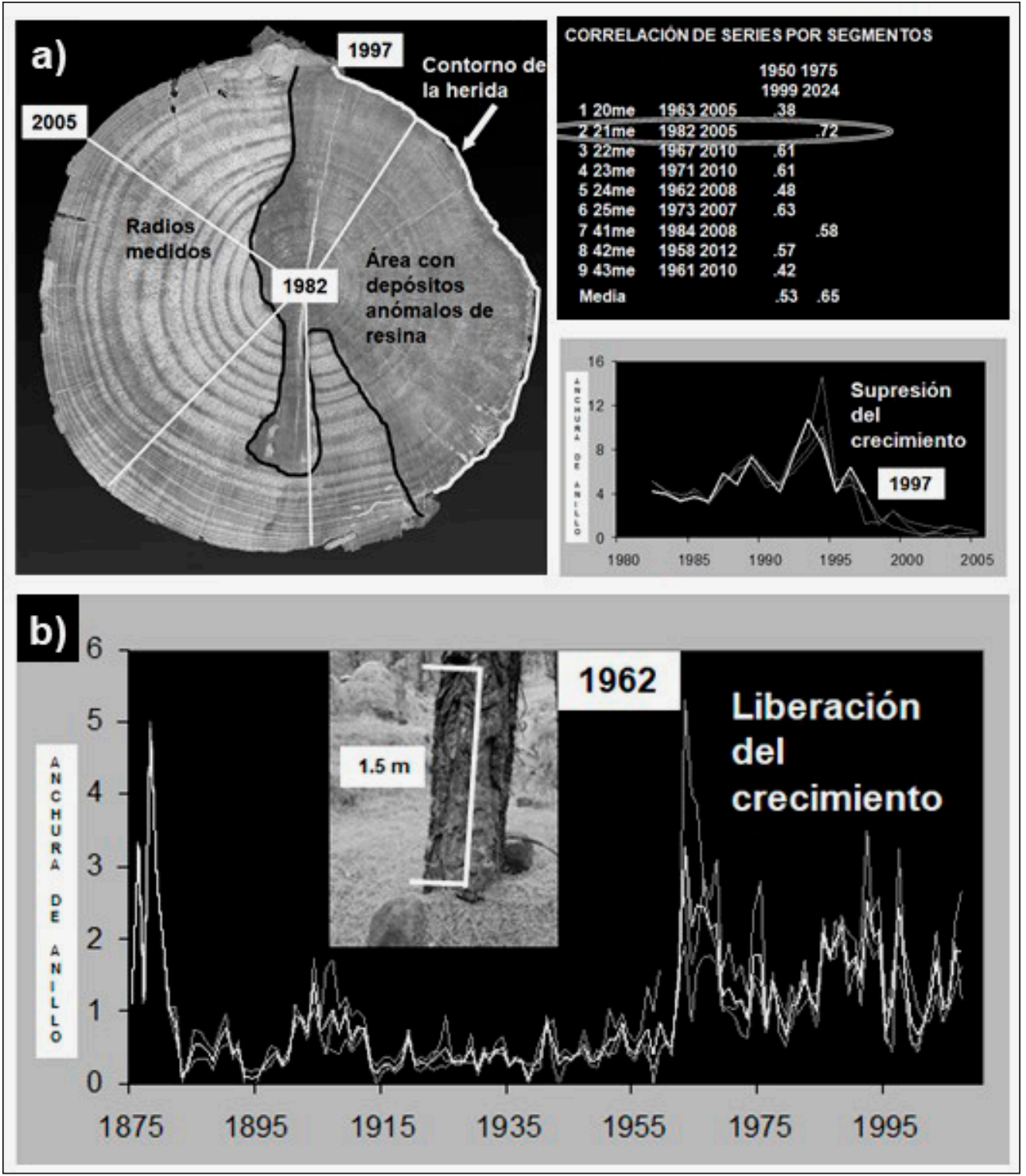

Figura 6. Ejemplos de respuesta de los árboles al daño en la Caldera de Taburiente: a) supresión del crecimiento que finalizó con la muerte del árbol: sección transversal que muestra los depósitos resinosos anómalos (izquierda), índice de correlación con los árboles próximos (derecha y arriba) y secuencias de anillos en los radios medidos (derecha abajo). b) Liberación del crecimiento, comúnmente detectado en árboles viejos, después del daño de 1962 y una imagen que muestra un gran descortezado.

Fuente: Génova et al. (2015). 


\section{Arroyo Cabrera en Venero Claro}

La cuenca torrencial del Arroyo Cabrera está ubicada en la falda norte de la Sierra del Valle (Gredos oriental, Sistema Central español; 40 24'N; $4^{\circ} 39^{\prime} \mathrm{W}$ ), en el término municipal de Navaluenga (Ávila). Se trata de un torrente tributario del río Alberche (cuenca del Tajo) por su margen derecha, con carácter fluvio-torrencial de gran actividad, y formado por la confluencia de diversos arroyos. La cuenca vertiente del arroyo Cabrera tiene una extensión aproximada de $15,5 \mathrm{~km}^{2}$ y presenta una forma subtriangular. El desnivel máximo de la cuenca es de $1.188 \mathrm{~m}$, mientras que la longitud del cauce es de $5.500 \mathrm{~m}$, lo que define una pendiente media del 21,6\%.

Sus características físicas hacen que esta cuenca tenga un comportamiento torrencial con una rápida respuesta a eventos de precipitación intensos y prolongados. En particular, el 18 de diciembre de 1997 se produjo un evento torrencial de alta magnitud asociado a células de lluvia estacionarias que causaron fuertes lluvias, lo que provocó un deslizamiento de tierra poco profundo en la cabecera de la cuenca, que movilizó abundante material sólido en el canal. Como resultado, una inundación repentina muy cargada en sedimentos se dirigió río abajo, modificando la geomorfología fluvial y causando considerables daños a la vegetación adyacente, compuesta principalmente por coníferas mediterráneas y especies ribereñas.

En 2007, diez años después, se comenzaron a aplicar análisis dendrogeomorfológicos con el objetivo de calibrar las técnicas hasta el momento conocidas. En una primera etapa de análisis, se muestrearon diversas especies (Fraxinus angustifolia, Alnus glutinosa, Pinus pinaster, Populus spp. y Salix spp.) para identificar las diferentes señales anatómicas asociadas a dicho evento torrencial. Los análisis mostraron que las señales son dependientes de la especie y de la edad y el estado vital del árbol (Ballesteros-Cánovas et al., 2010a, 2010b, 2015b). Posteriormente, se llevó a cabo una reconstrucción de la magnitud del evento en un tramo que concentraba una importante cantidad de evidencias botánicas definiendo indicadores de paleonivel. La aplicación de modelos hidráulicos bidimensionales sobre una topografía de alta precisión facilitó una reconstrucción del máximo caudal de la avenida con un elevado grado de fiabilidad. Dicho evento fue cuantificado en $79 \pm 14 \mathrm{~m} 3 \mathrm{~s}^{-1}$, lo que correspondía a una probabilidad anual de excedencia de 0,02 de acuerdo con datos existentes (Ballesteros-Cánovas et al., 2011a).

Actualmente, en la zona se están llevando a cabo análisis liquenométricos con el objetivo de tratar de caracterizar temporalmente una serie de depósitos de bloques y cantos asociados a eventos de avenida torrencial y flujos de derrubios, que serán también asociados a estimaciones de caudal determinadas mediante dos aproximaciones diferentes: por un lado, la aplicación de formulaciones de paleo-competencia de transporte y, por otro, la modelización hidráulica de caudales calibrada mediante la posición en X-Y-Z de los líquenes muestreados. El objetivo final será la caracterización y mejora del análisis de frecuencia de avenidas mediante la utilización de datos sistemáticos (serie temporal de diecisiete años de duración obtenida mediante limnímetros de presión y rádar de pulsos) y datos no sistemáticos (dendrogeomorfológicos y liquenométricos).

Se ha obtenido la curva de crecimiento del liquen reloj, Rhizocarpon geographicum, a partir de las medidas de los cementerios en las localidades circundantes (Navaluenga, el Tiemblo, El Barraco, Burgohondo y San Martin de Valdeiglesias), en los que se han tomado medidas sobre las superficies horizontales de tumbas cuyas edades están comprendidas entre 1911 y 2008 (figura 7). 


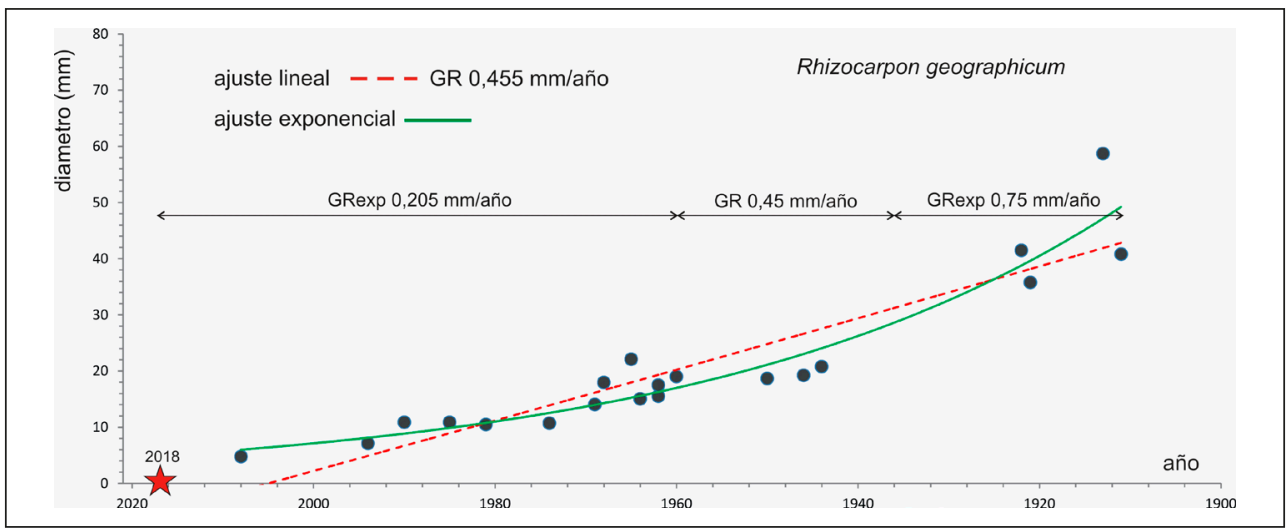

Figura 7. Curva de crecimiento de Rhizocarpon geographicum obtenida en varios cementerios cercanos a la cuenca de Venero Claro. Ajuste lineal de 0,455 mm/año. Ajuste exponencial (GRexp), dividido en tramos de crecimiento según su pendiente media. La estrella sobre 2018 en el eje de abscisas indica el año de la medida de los diámetros sobre las sepulturas de los cementerios. El ajuste exponencial presenta diferentes tasas de crecimiento anual según el intervalo de medida seleccionado. Se han dividido en tres tramos principales según las principales variaciones de pendiente de la curva.

En paralelo se han medido un total de 148 diámetros sobre líquenes de la misma especie Rhizocarpon geographicum, localizados sobre diversos bolos de granito del lóbulo superior del campo de bolos de Venero Claro, formado por depósito tras eventos torrenciales de avenidas e inundaciones. A partir de estos valores, se ha obtenido la edad de cada liquen aplicando el valor de crecimiento anual obtenido con el ajuste lineal de $\mathrm{GR}=0,455 \mathrm{~mm} /$ año (figura 7). Este valor está en consonancia con el obtenido por Bradwell y Armstrong (2006) de 0,67 mm/año, en Islandia, a partir de una medida sistemática de cinco años sobre bolos de morrenas glaciares. La diferencia de clima entre ambos lugares explicaría la divergencia entre ambos valores.

El error asociado a cada medida es de \pm 4 años, obtenido a partir de los valores extremos del ajuste exponencial $(0,20-0,75 \mathrm{~mm})$. No se han evaluado los periodos de colonización del liquen sobre los bolos de granito (periodo de ecesis). Se observa (figura 8), que el máximo de frecuencia de líquenes sobre los bolos aparece en el año de $1996 \pm$ 4, veintidós años antes de la fecha de medida (2018). Esta fecha coincide con la gran avenida de diciembre de 1997, que movilizó gran parte de la carga sólida presente actualmente en el campo de bolos. Además, se observa también cierta coincidencia entre los valores de los años húmedos con la frecuencia máxima de edades de líquenes sobre la roca. Aunque se aprecia un desplazamiento entre ambas curvas, que corresponde al error de obtención de la edad liquen y al periodo de ecesis o de colonización del Rhizocarpon sobre los bolos.

Resulta interesante que, a partir de este valor de crecimiento, se puede extrapolar la función hacia atrás en el tiempo para estimar la edad de otros depósitos de bolos más antiguos que hayan sido movilizados en eventos que hayan ocurrido antes del periodo instrumental y de los que no se disponen datos histórico-documentales, con el fin de hacer un estudio sobre periodos de retorno con, al menos, datos del siglo xix. 


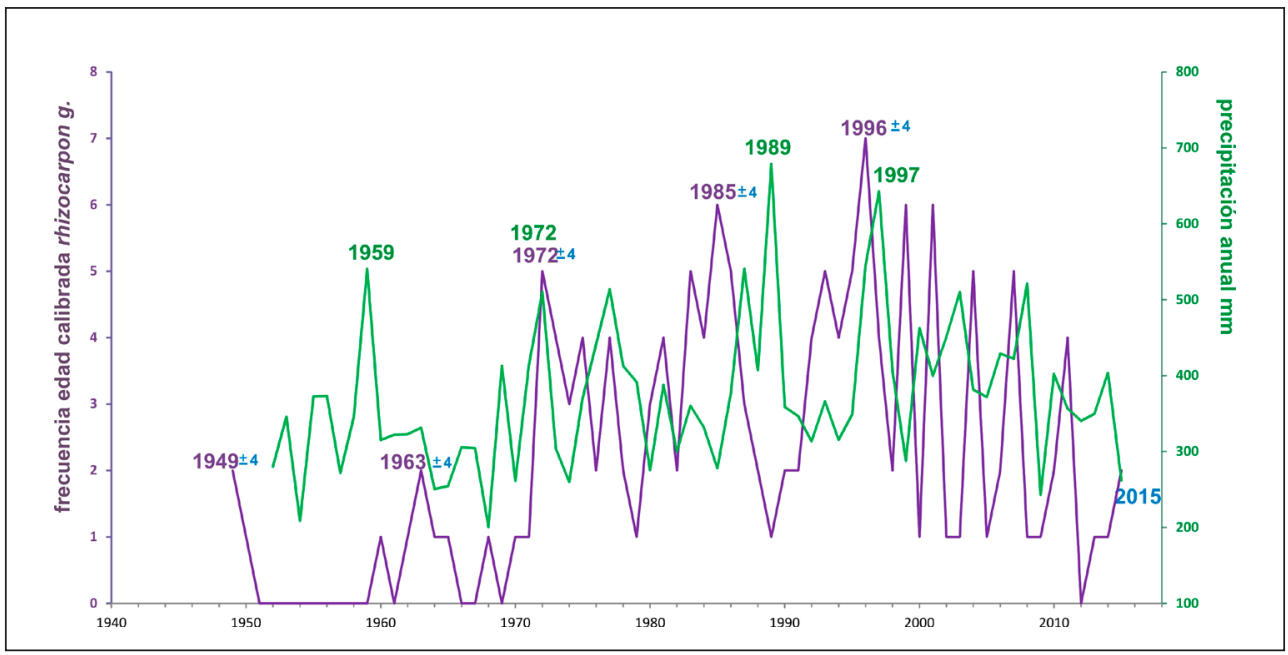

Figura 8. Frecuencias del diámetro de talos Rhizocarpon geographicum sobre los bolos del lóbulo superior del depósito y precipitación anual $(\mathrm{mm})$ en la comarca. Eje de ordenadas de la izquierda: valores de frecuencia de edades de líquenes medidos sobre el lóbulo superior del campo de bolos de Venero Claro; las edades presentan una error de \pm 4 años (ver interpretación en el texto). Eje de ordenadas de la derecha: valores de precipitaciones medias anuales de la estación meteorológica Ávila de la AEMET (www.aemet.es) entre 1952 y 2021 (representado hasta 2015), obtenidos con fecha junio 2021.

\section{El barranco de Portainé (Pirineo de Lleida)}

La cuenca del Portainé, en cuya cabecera se sitúa la estación de esquí de Port Ainé (Lleida, Pirineo catalán), presenta fenómenos de erosión y sedimentación activos provocados por frecuentes inundaciones torrenciales. Estas inundaciones presentan un gran riesgo potencial e incurren en pérdidas económicas importantes y, como sucede en tantas cuencas hidrográficas de montaña, prácticamente no se cuenta con registros de datos hidrológicos y climáticos. En Génova et al. (2018) estimamos la distribución temporal y espacial de las inundaciones torrenciales mediante técnicas dendrogeomorfológicas, con el objetivo de conocer si las actividades antrópicas (cambios de uso del suelo y obras de infraestructura) afectaban a su frecuencia y magnitud. Para ello, se analizaron ciento sesenta y seis muestras de sesenta y siete árboles pertenecientes a diez especies diferentes. Se identificaron los eventos de inundaciones pasados de los últimos cincuenta años mediante el análisis de diferentes tipos de evidencias: callos y cicatrices internas caracterizadas por la desestructuración de los tejidos; decapitación y sustitución del eje principal por una o más ramas laterales (figura 9); asimetrías en las series de anillos de árboles o cambios bruscos en la anchura de los anillos. También se realizó un estudio geomorfológico detallado del área analizada y se recopilaron los datos históricos disponibles. Además, el análisis concreto de la altura de los daños en los árboles producidos en 2008 y 2010, junto con la 
realización de una topografía muy detallada y la elaboración de un modelo hidráulico unidimensional, ha permitido obtener una estimación de los caudales y reconocer que los datos procedentes de árboles situados en áreas con procesos torrenciales de energía intermedia son los más confiables para estimar las descargas máximas (Victoriano et al., 2018). La comprensión holística de esta compleja cuenca hidrográfica, que solo puede ser descrita, caracterizada y analizada mediante una investigación multidisciplinar (Furdada et al., 2020), ha proporcionado una nueva perspectiva sobre los cambios

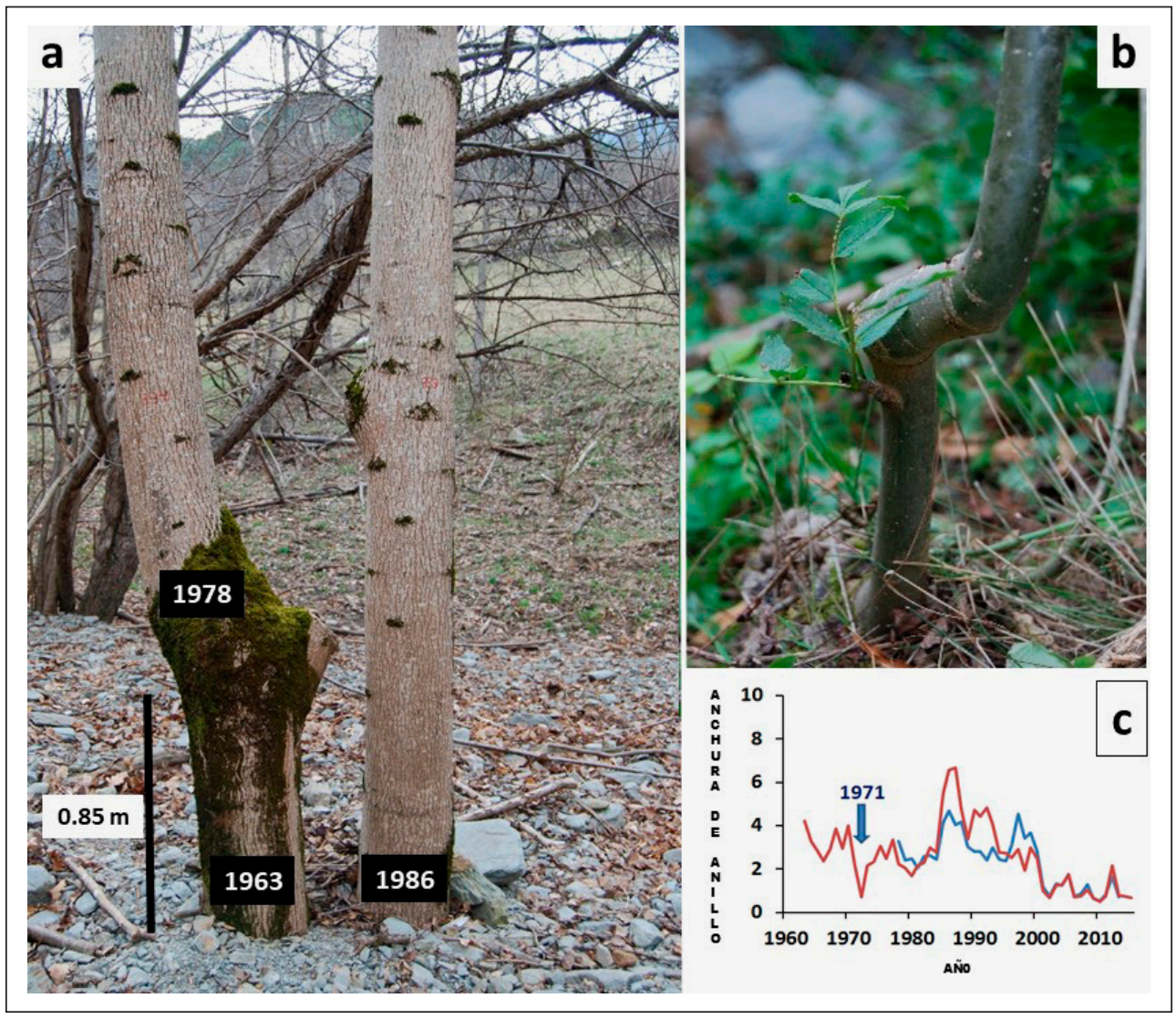

Figura 9. Fresnos norteños o de hoja ancha (Fraxinus excelsior L.) en el barranco de Portainé. a) Árbol PA33 (a la izquierda) que fue decapitado por un evento que se estima que ocurrió en el año dendrogeomorfológico 1969-1970 y árbol PA77 (a su derecha) que germinó más tarde. b) Un árbol muy joven decapitado que muestra una rama de remplazo. c) Series de anchura de los anillos $(\mathrm{mm})$ para el árbol PA33; la correspondiente al tronco principal es la línea de color rojo (que también indica la supresión de crecimiento detectada en 1971), mientras que la línea azul corresponde a la rama de remplazo, que comienza en 1978.

Fuente: Génova et al. (2018). 
en la frecuencia de las inundaciones desde 2006, y muy especialmente desde 2008, sugiriendo que el equilibrio geomorfológico y la hidrología superficial de la cuenca se han alterado, coincidiendo tanto con grandes movimientos de tierra en la estación de esquí como con lluvias intensas pero no extraordinarias y produciendo un cambio en la dinámica torrencial de la cuenca que se transformó en flujos muy densos y cargados de sedimentos altamente erosivos (Génova et al., 2018; Furdada et al., 2020).

\section{Discusión}

Las técnicas dendrogeomorfológicas y liquenométricas presentan importantes ventajas y aportaciones al estudio de los sistemas fluviales, como ha quedado demostrado en los casos prácticos indicados. Estas técnicas resultan un complemento a los métodos clásicos (hidrológico-hidráulicos e históricodocumentales) y paleohidrológicos novedosos (geológico-geomorfológicos) en sistemas fluviales con información diversa, donde la integración de diferentes fuentes de datos resulta fundamental para entender los cambios ambientales (Schulte et al., 2019; Furdada et al., 2020).

Entre las principales ventajas y aportaciones de la dendrogeomorfología y la liquenometría en el estudio de fenómenos de avenida en sistemas fluviales torrenciales se encuentra aportar información sobre la frecuencia y magnitud de los eventos extremos allí donde no existen otro tipo de datos (por falta de información instrumental sistemática o documental histórica), o donde los registros existentes son cortos, poco confiables y/o discontinuos en espacio y tiempo. Estas zonas montañosas, muchas veces alejadas de los núcleos de población y, por lo tanto, de los sistemas instrumentales y centros de producción documental, en ocasiones solo contienen un registro natural de los eventos de avenidas torrenciales; y, ante la imposibilidad de datar las formas y los depósitos fluviales, las fuentes de datos botánicas nos aportan la única información cuantitativa sobre el fenómeno torrencial.

Sin embargo, también presentan limitaciones en la aplicación y validez de sus resultados, las más importantes las detallamos a continuación.

\section{Limitaciones del análisis dendrogeomorfológico en el estudio de avenidas torrenciales}

Las limitaciones del uso de la dendrogeomorfología en el estudio de avenidas torrenciales se deben fundamentalmente a: $(i)$ la disponibilidad de árboles y/o arbustos susceptibles de ser analizados dendrocronológicamente, (ii) identificación e interpretación de la respuesta fisiológica del árbol en el registro de anillos de crecimiento a cambios ambientales, (iii) incertidumbres asociadas a las marcas de paleo estado (p. ej., la altura de descortezados o inclinación de árboles). Respecto a la disponibilidad de individuos, a pesar de la ubicuidad de especies vegetales en la mayoría de las zonas de montaña del mundo (excepto en zonas desérticas y en pisos nivales), los individuos localizados en zonas tropicales y subtropicales presentan una mayor problemática para su datación cruzada. El análisis dendrocronológico de árboles en estas zonas se circunscribe a áreas de montaña, donde los factores limitantes de crecimiento pueden estar más marcados (Bodoque et al., 2015). Además, en muchos casos, la datación cruzada de estos individuos requerirá metodologías añadidas, como lo es el ${ }^{14} \mathrm{C}$ (Brienen et al., 2016) o análisis anatómicos (Quesada-Román et al., 2020b). Por otro lado, se sabe que la respuesta fisiológica 
de los árboles y arbustos depende de la especie (Ballesteros et al., 2010b; Silhán y Stoffel, 2015), la edad, el estado fitosanitario previo y el clima (Ballesteros-Cánovas et al., 2015b). Esto dificulta que puedan aplicarse criterios fijos a la hora de definir las perturbaciones de crecimiento, fundamentalmente en relación con la intensidad de las señales, ya que, en gran medida, son específicas del individuo y su hábitat. Además, desde un punto de vista geomorfológico, la interpretación de la datación dendrogeomorfológica de eventos de inundaciones se encuentra limitada por la propia dinámica fluvial. El grado de impacto de las crecidas repentinas registradas en los árboles está relacionado con su posición geográfica y edad, pero no existe necesariamente una correlación directa entre las fechas de los mayores eventos de flujo y la cantidad e intensidad de la evidencia dendrogeomorfológica (Ruiz-Villanueva et al., 2010). De hecho, los eventos que se registran mejor son los de magnitud intermedia, pues los de mayor envergadura pueden destruir toda la vegetación y los de menor magnitud dejan pocas evidencias (Ruiz-Villanueva et al., 2010; Génova et al., 2015). Además, los efectos de eventos sucesivos pueden quedar enmascarados ya que, por ejemplo, un suceso posterior de mayor magnitud destruiría toda la evidencia dendrogeomorfológica de un suceso anterior menos intenso (Ballesteros-Cánovas et al., 2013) y los daños más recientes (que ocurrieron hace uno o dos años) son muy difíciles de fechar porque aún no se pueden identificar los anillos del callo adecuadamente (Génova et al., 2018). Por último, existen limitaciones relacionadas con la definición de marcas de paleo-estado, que en gran medida dependen de la dinámica fluvial y transporte de sedimentos (Ballesteros et al., 2011b). En relación con los descortezados, se ha observado que las alturas máximas de los mismos con marcas de aguas altas son comparables (Gottesfeld, 1996), aunque dependan de la ubicación del árbol en relación con la dinámica fluvial (Ballesteros-Canovas et al., 2015a, 2016; Victoriano et al., 2018; Quesada-Roman et al., 2020b). A pesar de ello, las aproximaciones del registro dendrogeomorfológico a la recurrencia y extensión de las avenidas torrenciales resultan sumamente interesantes, especialmente para incrementar la información de los registros disponibles o cuando éstos no existen.

\section{Limitaciones del análisis liquenométrico en el estudio de avenidas torrenciales}

La principal limitación de la aplicación de la liquenometría al análisis de las avenidas es la escasez general de estudios liquenométricos previos en ambientes fluviales torrenciales, lo que supone un vacío de investigación que impide tener referentes en los que basar o fundamentar trabajos posteriores. En este sentido, el presente artículo persigue recopilar y poner a disposición de la comunidad científica los escasos estudios que pueden servir de referencia en el futuro a los investigadores de los medios torrenciales.

Entre las principales limitaciones metodológicas de la liquenometría se encuentran las determinaciones taxonómicas de los líquenes que emplea para la datación de los cambios ambientales. El problema en la utilización mayoritaria del género Rhizocarpon en liquenometría es que dicho género de liquen consta de más de doscientas especies y subespecies (Roca-Valiente et al., 2016), con lo que constituye un grupo polifilético. Todo ello provoca que actualmente difiera su descripción y taxonomía 
por diferentes autores, y son insuficientes las claves de identificación actuales para dirimir tal divergencia filética.

Además, un importante aspecto que se deben tener en cuenta en la aplicación de la liquenometría es la determinación del periodo de colonización (ecesis) de la especie reloj y su relación con el periodo climático dominante en el periodo de datación. En general, los periodos de colonización oscilan de pocos meses a pocos años (Pérez-López et al., 2019). Por ello, es conveniente realizar un estudio de la frecuencia de especies de líquenes por diámetros y los periodos climáticos diferenciados por su dominio seco o húmedo, o bien por los índices de lluvia.

\section{Conclusión}

Las fuentes de datos y las metodologías botánicas para la detección de cambios ambientales en sistemas fluviales, como la dendrogeomorfología y la liquenometría, a pesar de sus limitaciones, presentan unas ventajas y aportaciones incuestionables en el estudio de las avenidas torrenciales. Entre las ventajas se encuentran su ubicuidad en sistemas fluviales torrenciales, frente a la ausencia de otras fuentes de información (instrumentales o documentales). Entre los inconvenientes, la escasa proliferación de este tipo de estudios, tanto internacionalmente como en España; y dificultades metodológicas inherentes a la dendrocronología y la liquenología. Por ello, estas técnicas resultan un complemento a los métodos clásicos en sistemas fluviales con información diversa. Los estudios de aplicación en España, aunque todavía escasos, suponen iniciativas pioneras incluso a nivel internacional y pueden servir como ejemplos para su extensión a otros territorios y problemáticas. Porque en ocasiones, en sistemas fluviales sin apenas información convencional, la dendrogeomorfología y liquenometría son la única alternativa para conocer la frecuencia y magnitud de los cambios ambientales ligados a las avenidas e inundaciones.

\section{Agradecimientos y FUENTES DE FINANCIACIÓN}

Los autores desean agradecer su colaboración a los equipos humanos de los proyectos de investigación que han financiado los trabajos sobre estas temáticas en la última década y media en España (GeoRiada, Dendro-Avenidas, MAS Dendro-Avenidas, IDEA-GesPPNN, MARCoNI, CHARMA y DRAINAGE); y especialmente al Dr. José María Bodoque del Pozo (UCLM), la Dra. Virginia Ruiz Villanueva (U. de Lausanne), la Dra. Gloria Furdada (UB) y la Dra. Ane Victoriano. Este artículo forma parte de los trabajos de recopilación y discusión de los métodos paleohidrológicos (GT1.T1) del proyecto "Metodologías avanzadas para el análisis científico-técnico del riesgo por inundaciones para la mejora de la resiliencia y la reducción del riesgo" (DRAINAGE-3-R), del Plan Nacional de I+D+i, convocatoria Retos (CGL2017-83546-C3-3-R, MINEICO/AEI/FEDER, UE). 


\section{REFERENCIAS}

Alestalo, J. (1971). Dendrochronological interpretation of geomorphic processes. Fennia, 105, 1-140.

Aloni, R. (2015). Ecophysiological implications of vascular differentiation and plant evolution. Trees, 29(1), 1-16.

Amstrong, R.A. (2016). Lichenometric dating (lichenometry) and the biology of the lichen genus Rhizocarpon: challenges and future directions. Geografiska Annaler: Series A, Physical Geography, 98(3), 183-206. doi:10.1111/geoa.12130.

Aristizábal, E., Arango Carmona, M. I., \& García López, I. K. (2020). Definición y clasificación de las avenidas torrenciales y su impacto en los Andes colombianos. Cuadernos de Geografía: Revista Colombiana de Geografía, 29(1), 242-258.

Baker, V. R. (2008). Paleoflood hydrology: Origin, progress, prospects. Geomorphology, 101(1-2), 1-13. Ballesteros-Cánovas, J. A., Trappmann, D., Shekhar, M., Bhattacharyya, A., \& Stoffel, M. (2017a). Regional flood-frequency reconstruction for Kullu district, Western Indian Himalayas. Journal of Hydrology, 546, 140-149.

Ballesteros-Cánovas, J. A. \& Stoffel, M. (2016). Biologic Dating Techniques. En Richardson, D., Castree, N., Goodchild, M. F., Kobayashi, A., Liu, W., \& Marston, R. A. (eds.). International Encyclopedia of Geography: People, the Earth, Environment and Technology (vol. 15, pp. 1-7). Wiley. doi: 10.1002/9781118786352.wbieg0806

Ballesteros-Cánovas, J. A., Bodoque, J. M., Díez, A., Sánchez-Silva, M., \& Stoffel, M. (2011b). Calibration of floodplain roughness and estimation of palaeoflood discharge based on tree-ring evidence and hydraulic modelling. Journal of Hydrology, 403(1-2), 103-115.

Ballesteros-Cánovas, J. A., Stoffel, M., Bodoque, J. M., Bollschweiler, M., Hitz, O., \& Díez-Herrero, A. (2010b). Wood anatomy of Pinus pinaster Ait. following wounding by flash floods. Tree-Ring Research, 66(2), 93-103.

Ballesteros-Cánovas, J. A., Stoffel, M., Martín-Duque, J. F., Corona, C., Lucía, A., Bodoque, J. M., \& Montgomery, D. R. (2017b). Gully evolution and geomorphic adjustments of badlands to reforestation. Nature Scientific Reports, 7, 45027.

Ballesteros-Cánovas, J. A., Stoffel, M., St George, S., \& Hirschboeck, K. (2015a). Flood records in tree rings: a review. Progress and Physical Geography, 39(6), 794-816.

Ballesteros-Cánovas, J. A., Stoffel, M., Benito, G., Rohrer, M., Barriopedro, D., García-Herrera, R., \& Brönnimann, S. (2018). On the extraordinary winter flood episode over the North Atlantic Basin in 1936. Annals of the New York Academy of Sciences, 1436(1), 206-216.

Ballesteros-Cánovas, J. A., Stoffel, M., \& Guardiola Albert, C. (2015b). XRCT imagery and variograms reveal 3D changes in wood density of riparian trees affected by floods. Trees, 29, 1115-1126.

Ballesteros-Cánovas, J. A., Eguibar, M., Bodoque, J. M., Díez-Herrero, A., Stoffel, M., \& GutierrezPérez, I. (2011a). Estimating flash flood discharge in an ungauged mountain catchment with 2D hydraulic models and dendrogeomorphic paleostage indicators. Hydrological Processes, 25, 970-979. 
Ballesteros-Cánovas, J. A., Márquez-Peñaranda, J. F., Sánchez-Silva, M., Díez-Herrero, A., RuizVillanueva, V., Bodoque, J. M., Eguibar, M. A., \& Stoffel, M. (2015c). Can tree tilting be used for paleoflood discharge estimations? Journal of Hydrology, 529, 480-489.

Ballesteros-Cánovas, J. A., Stoffel, M., Czajka, B., Janecka, K., Kaczka R. J., \& Lempa, M. (2016). Paleoflood discharge reconstruction in Tatra Mountain streams. Geomorphology, 272, 92-101.

Ballesteros-Cánovas, J. A., Stoffel, M., Bollschweiler, M., Bodoque, J. M., \& Díez-Herrero, A. (2010a). Flash-flood impacts cause changes in wood anatomy of Alnus glutinosa, Fraxinus angustifolia and Quercus pyrenaica. Tree Physiology, 30, 773-781.

Ballesteros-Cánovas, J. A. \& Álvarez-Troncoso, R. (2021). Riesgo y peligrosidad por avenidas súbitas aplicación de fuentes no sistémicas para su caracterización. Interreg Sudoe Inundation. European Regional Development Fund.

Ballesteros-Cánovas, J. A., Koul, T., Bashir, A., Bodoque, J. M., Allen, S., Guillet, S., Rashid, I., Alamgir, S. H., Shah, M., Bhat, M. S., Alam, A., \& Stoffel, M. (2020). Recent flood hazards in Kashmir put into context with millennium-long historical and tree-ring records. Science of The Total Environment, 722, 137875.

Ballesteros-Cánovas, J. A., Sanchez-Silva, M., Bodoque, J. M., \& Díez-Herrero, A. (2013). An integrated approach to flood risk management: a case study of Navaluenga (Central Spain). Water Resources Management, 27(8), 3051-3069.

Benito, G., Brázdil, R., Herget, J., \& Machado, M. J. (2015). Quantitative historical hydrology in Europe. Hydrology and Earth System Sciences, 19(8), 3517-3539.

Bodoque, J. M., Díez-Herrero, A., Eguibar, M. A., Benito, G., Ruiz-Villanueva, V., \& BallesterosCánovas, J. A. (2015). Challenges in paleoflood hydrology applied to risk analysis in mountainous watersheds - a review. Journal of Hydrology, 529, 449-467.

Bodoque, J. M., Ballesteros-Cánovas, J. A., \& Stoffel, M. (2020). An application-oriented specific protocol for flood frequency analysis from based on botanical evidence. Journal of Hydrology, 590, 125242. doi: $10.1016 / j . j$ hydrol.2020.125242

Bradwell, T. \& Armstrong, R. A. (2006). Growth rates of Rhizocarpon geographicum lichens: a review with new data from Iceland. Journal of Quaternary Science, 22(4), 311-320.

Bräuning, A., De Ridder, M., Zafirov, N., García-González, I., Dimitrov, D. P., \& Gärtner, H. (2016). Tree-ring features: indicators of extreme event impacts. IAWA Journal, 37(2), 206-231.

Brienen, R. J., Schöngart, J., \& Zuidema, P.A. (2016). Tree rings in the tropics: insights into the ecology and climate sensitivity of tropical trees. Tropical tree physiology, 439-461.

Copini, P., Decuyper, M., Sass-Klaassen, U., Gärtner, H., Mohren, F., \& den Ouden, J. (2015). Effects of experimental stem burial on radial growth and wood anatomy of pedunculate oak. Dendrochronologia, 33, 54-60.

De Micco, V., Campelo, F., De Luis, M., Bräuning, A., Grabner, M., Battipaglia, G., \& Cherubini, P. (2016). Intra-annual density fluctuations in tree rings: how, when, where, and why? IAWA Journal, $37(2), 232-259$. 
Díez-Herrero, A., Ballesteros-Cánovas, J. A., Bodoque, J. M., Eguibar, M. A., Fernandez, J. A., Genova, M. M., \& Stoffel, M. (2007). Improving flood frequency and magnitude estimates using dendrogeomorphological techniques. Boletin Geologico y Minero, 118(4), 789-802.

Díez-Herrero, A., Ballesteros-Cánovas, J. A., Ruiz-Villanueva, V., \& Bodoque J. M. (2013a). A review of dendrogeomorphological research applied to flood risk analysis in Spain. Geomorphology, 196, 211-220.

Díez-Herrero, A., Ballesteros-Cánovas, J. A., Bodoque, J. M., \& Ruiz-Villanueva, V. (2013b). A new methodological protocol for the use of dendrogeomorphological data in flood risk analysis. Hydrology Research, 44(2), 234-247.

Díez-Herrero, A. (2021). La dendrogeomorfología como fuente de datos de la frecuencia y magnitud de caudales históricos. En Jiménez, A. (ed.). Paleocrecidas y avenidas históricas y su aplicación a la seguridad hidrológica de las presas (monografías CEDEX, cap. III, pp. 61-92). Madrid: Centro de Estudios y Experimentación de Obras Públicas.

Díez-Herrero, A. \& García Rodríguez, M. (2016). Riesgos por avenidas e inundaciones. En Lario, J. \& Bardají, T. (coords.). Introducción a los Riesgos Geológicos (pp. 119-152). Madrid: UNED.

Douglass, A. E. (1941). Crossdating in dendrochronology. Journal of Forestry, 39(10), 825-831.

Ferrio, J. P., Díez-Herrero, A., Tarrés, D., Ballesteros-Cánovas, J. A., Aguilera, M. \& Bodoque, J. M. (2015). Using stable isotopes of oxygen from tree-rings to study the origin of past flood events: First results from the Iberian Peninsula. Quaternaire. Revue de l'Association française pour l'étude du Quaternaire, 26(1), 67-80.

Fontana, G. A., Egli, M., \& Gärtner, H. (2021). Testing the potential of the dwarf shrub Dryas octopetala L. for dating in dendrogeomorphology. Dendrochronologia, 67, 125823.

Friedman, J. M., Vincent, K. R., \& Shafroth, P. B. (2005). Dating floodplain sediments using tree-ring response to burial. Earth Surface Processes and Landforms, 30(9), 1077-1091.

Fritts, H. C. (1976). Tree Ring and Climate. Londres: Academic Press.

Furdada, G., Victoriano, A., Díez-Herrero, A., Génova, M., Guinau, M., De las Heras, A., Palau, R. M., Hürlimann, M., Khazaradze, G., Casas, J. M., Margalef, A., Pinyol, J., \& González, M. (2020). Flood consequences of land-use changes at a ski resort: Overcoming a geomorphological threshold (Portainé, Eastern Pyrenees, Iberian Peninsula). Water, 12(2), 368.

Garrote, J., Díez-Herrero, A., Bodoque, J. M., Perucha, M. A., Mayer, P., \& Genova, M. (2018a). Flood hazard management in public mountain recreation areas vs. ungauged fluvial basins. The example of Caldera de Taburiente National Park, Canary Islands (Spain). Geosciences, 8(6), 1-23. doi:10.3390/geosciences 8010006

Garrote, J., Díez-Herrero, A., Génova, M., Bodoque, J. M., Perucha, M. A., \& Mayer, P. L. (2018b). Improving flood maps in ungauged fluvial basins with dendrogeomorphological data. An example from the Caldera de Taburiente National Park (Canary Islands, Spain). Geosciences, 8(300), 1-18. doi: $10.3390 /$ geosciences 8080300 
Génova, M., Máyer, P., Ballesteros-Cánovas, J. A., Rubiales, J. M., Saz, M. A., \& Díez-Herrero, A. (2015). Multidisciplinary study of flash floods in the Caldera de Taburiente National Park (Canary Islands, Spain). Catena, 131, 22-34.

Génova, M., Díez-Herrero, A., Furdada, G., Guinau, M., \& Victoriano, A. (2018). Dendrogeomorphological evidence of flood frequency changes and human activities (Portainé Basin, Spanish Pyrenees). Tree-ring Research, 74(2), 144-161.

Gob, F., Petit, F., Bravard, J.-P., Ozer, A., \& Gob, A. (2003). Lichenometric application to historical and subrecent dynamics and sediment transport of a Corsican stream (Figarella River-France). Quaternary Science Reviews, 22(20), 2111-2124. doi:10.1016/S0277-3791(03)00142-2

Gottesfeld, A. S. (1996). British Columbia flood scars: maximum flood-stage indicators. Geomorphology, 14(4), 319-325.

Innes, J. L. (1986). Dating Exposed Rock Surfaces in the Arctic by Lichenometry: The Problem of Thallus Circularity and Its Effect on Measurement Errors. Artic, 39(3), 253-259.

Malik, I. (2008). Dating of small gully formation and establishing erosion rates in old gullies under forest by means of anatomical changes in exposed tree roots (Southern Poland). Geomorphology, 93(3-4), 421-436.

Marchi, L., Borga, M., Preciso, E., \& Gaume, E. (2010). Characterisation of selected extreme flash floods in Europe and implications for flood risk management. Journal of Hydrology, 394(1-2), $118-133$

Mayer, B., Stoffel, M., Bollschweiler, M., Hübl, J., \& Rudolf-Miklau, F. (2010). Frequency and spread of debris floods on fans: a dendrogeomorphic case study from a dolomite catchment in the Austrian Alps. Geomorphology, 118(1-2), 199-206.

Nanson, G. C. \& Beach, H. F. (1977). Forest succession and sedimentation on a meandering-river floodplain, northeast British Columbia, Canada. Journal of Biogeography, 4(3), 229-251.

Novak, K., de Luis, M., Saz M. A., Longares, L. A., Serrano-Notivoli, R., Raventós, J., Čufar, K., Gričar, J., Di Filippo, A., Piovesan, G., Rathgeber, C. B. K., Papadopoulos, A., \& Smith, K. T. (2016a). Missing Rings in Pinus halepensis - The Missing Link to Relate the Tree-Ring Record to Extreme Climatic Events. Frontiers in Plant Science, 7, 727.

Novak, K., de Luis, M., Gričar, J., Prislan, P., Merela, M., Smith, K. T., \& Čufar, K. (2016b). Missing and dark rings associated with drought in Pinus halepensis. IAWA Journal, 37, 260-274.

Novak, K., Saz Sánchez, M. A., Čufar, K., Raventós, J., \& de Luis, M. (2013). Age, climate and intra-annual density fluctuations in Pinus halepensis in Spain. IAWA Journal, 34, 459-474.

Osborn, G., McCarthy, D., LaBrie, A., \& Burke, R. (2015). Lichenometric dating: Science or pseudoscience? Quaternary Research, 83, 1-12.

Pérez-López, R., Martín-González, F., Martínez-Díaz, J. J., \& Rodríguez-Pascua, M. A. (2012). Datación mediante liquenometría de los desprendimientos rocosos asociados a la sismicidad histórica en Lorca. Boletín Geológico y Minero, 123(4), 473-485.

Pérez-López, R., Giner-Robles, J. L., Rodríguez-Pascua, M. A., Silva, P. G., Roquero, E., Bardají, T., Elez, J., \& Huerta, P. (2019). Lichenometric dating of coseismic rockfall related to the Great Lisbon 
Earthquake in 1755 affecting the archaeological site of "Tolmo de Minateda" (Spain). Zeitschrift für Geomorphologie, 62(2), 271-293. https://doi.org/10.1127/zfg_suppl/2019/0504

Quesada-Román, A., Ballesteros-Cánovas, J. A., Granados, S., Birkel, C., \& Stoffel, M. (2020a).

Dendrogeomorphic reconstruction of floods in a dynamic tropical river. Geomorphology, 359, 107133.

Quesada-Román, A., Ballesteros-Cánovas, J. A., Guillet, S., Madrigal-González, J., \& Stoffel, M. (2020b). Neotropical Hypericum irazuense shrubs reveal recent ENSO variability in Costa Rican páramo. Dendrochronologia, 61, 125704.

Roca-Valiente, B., Hawksworth, D., Pérez-Ortega, S., Sancho, L., \& Crespo, A. (2016). Type studies in the Rhizocarpon geographicum group (Rhizocarpaceae, lichenized Ascomycota). The Lichenologist, 48(2), 97-110. doi:10.1017/S002428291500050X

Rosenwinkel, S., Korupa, O., Landgraf, A., \& Dzhumabaeva, A. (2015). Limits of lichenometry. Quaternary Science Reviews, 129, 229-238.

Ruiz-Villanueva, V., Díez-Herrero, A., Stoffel, M., Bollschweiler, M., Bodoque, J. M., \& BallesterosCánovas, J. A. (2010). Dendrogeomorphic analysis of flash floods in a small ungauged mountain catchment (Central Spain). Geomorphology, 118(3-4), 383-392.

Schulte, L., Wetter, O., Wilhelm, B., Peña, J. C., Amann, B., Wirth, S. B., Carvalho, F., \& GómezBolea, A. (2019). Integration of multi-archive datasets for the development of a four-dimensional paleoflood model of alpine catchments. Global and Planetary Change, 180, 66-88.

Schweingruber, F. H. (2007). Wood structure and environment. Springer Science \& Business Media.

Schweingruber, F. H. (2012). Tree rings: basics and applications of dendrochronology. Springer Science \& Business Media.

Shigo, A. L. (1984). Compartmentalization: a conceptual framework for understanding how trees grow and defend themselves. Annual review of phytopathology, 22(1), 189-214.

Sigafoos, R. S. (1961). Vegetation in relation to flood frequency near Washington, DC. US Geological Survey Professional Paper, 424-C, 248-249.

Sigafoos, R. S. (1964). Botanical evidence of floods and flood-plain deposition. US Geological Survey Professional Paper, 485-A, 1-35.

Šilhán, K. \& Stoffel, M. (2015). Impacts of age-dependent tree sensitivity and dating approaches on dendrogeomorphic time series of landslides. Geomorphology, 236, 34-43.

Stoffel, M., Bollschweiler, M., Butler, D. R., \& Luckman, B. H. (2010a). Tree rings and natural hazards: an introduction. En Tree Rings and Natural Hazards (pp. 3-23). Dordrecht: Springer.

Stoffel, M., Bollschweiler, M., Butler, D. R., \& Luckman, B. H. (eds.). (2010b). Tree rings and natural hazards: a state-of-art (vol. 41). Springer Science \& Business Media.

Therrell, M. D. \& Bialecki, M. B, (2014). A multi-century tree-ring record of spring flooding on the Mississippi River. Journal of Hydrology, 592, 490-498.

Tichavský, R., Koutroulis, A., Chalupová, O., Chalupa, V., \& Šilhán, K. (2020). Flash flood reconstruction in the Eastern Mediterranean: Regional tree ring-based chronology and assessment of climate triggers on the island of Crete. Journal of Arid Environments, 177, 104135. 
Timell, T. E. (1986). Compression Wood in Gymnosperms. Berlín: Spinger-Verlag.

Victoriano, A., Díez-Herrero, A., Génova, M., Guinau, M., Furdada, G., Khazaradze, G., \& Calvet, J. (2018). Four-topic correlation between flood dendrogeomorphological evidence and hydraulic parameters (the Portainé stream, Iberian Peninsula). Catena, 162, 216-229.

Wertz, E. L., St. George, S., \& Zeleznik, J. D. (2013). Vessel anomalies in Quercus macrocarpa tree rings associated with recent floods along the Red River of the North, United States. Water Resources Research, 49(1), 630-634.

Wilhelm, B., Ballesteros-Cánovas, J. A., Aznar, B., Kämpf, J. P. C., Swierczynski, T., Stoffel, M., Størenh, E., \& Toonen, W. (2018a). Recent advances in paleoflood hydrology: From new archives to data compilation and analysis. Water Security, 3, 1-8.

Wilhelm, B., Ballesteros-Cánovas, J. A., Macdonald, N., Toonen, W. H., Baker, V., Barriendos, M., Benito, G., Brauer, A., Corella, J. P., Denniston, R., Glaser, R., Ionita, M., Kahle, M., Liu, T., Luetscher, M., Macklin, M., Mudelsee, M., Munoz, S., Schulte, L., George, S. S., Stoffel, M., \& Wetter, O. (2018b). Interpreting historical, botanical, and geological evidence to aid preparations for future floods. WIRES, 6(1), e1318.

Zaginaev, V., Petrakov, D., Erokhin, S., Meleshko, A., Stoffel, M., \& Ballesteros-Cánovas, J. A. (2019). Geomorphic control on regional glacier lake outburst flood and debris flow activity over northern Tien Shan. Global and Planetary Change, 176, 50-59.

Zalloni, E., de Luis, M., Campelo, F., Novak, K., De Micco, V., Di Filippo, A., Vieira, J., Nabais, C., Rozas, V., \& Battipaglia, G. (2016). Climatic signals from intra-annual density fluctuation frequency in mediterranean pines at a regional scale. Frontiers in Plant Science, 7, 579.

Cómo citar este artículo:

Díez-Herrero, A., Ballesteros-Cánovas, J. A., Génova, M., Pérez-López, R., \& Garrote Revilla, J. (2021). Cambios ambientales detectados por dendrogeomorfología y liquenometría para el análisis de avenidas torrenciales en sistemas fluviales. Cuadernos de Geografía, 107, 93-121.

https://doi.org/10.7203/CGUV.107.21259

\section{(C) $(1) \Theta(\Theta$}

Este obra está bajo una licencia de Creative Commons Reconocimiento-NoComercial-SinObraDerivada 4.0 Internacional. 
